\title{
On the Field Theoretic Functional Calculus for the Anharmonic Oscillator III
}

\author{
W. Schuler and H. StumpF
}

Institut für Theoretische Physik der Universität Tübingen

(Z. Naturforsch. 23 a, 902-917 [1968] ; received 18 December 1967)

\begin{abstract}
The theory of solution for quantum field functional equations is developped for a suitable testproblem of quantum mechanics. In Sect. 1 the functional formulation of the anharmonic oscillator in its spinorial representation is given, and in Sect. 2 translational equivalent functional equations are discussed. The expansion of the physical state functionals into series of basefunctionals and the symmetrical representation of the functional equations for such an expansion is discussed in Sect. 3. In the following Sect. 4 the special symmetric orthogonal Hermitean functionals are used and the explicit representation is derived. In Sect. 5 the functionals are approximated by expansions with only a finite number of terms and the resulting equations are prepared for integration and in Sect. 6 a necessary condition of stationarity is considered. In Sect. 7 the simplest equation for $N=1$ is discussed in detail and the lowest eigenvalue is obtained. In the appendices technical details are derived.
\end{abstract}

In nonlinear spinor theory the dynamical behaviour of elementary particles can be described by functionals of field operators in a Heisenberg representation and corresponding functional equations ${ }^{1-3}$. To obtain the physical information, it is necessary to solve the functional equations without perturbation theory, i. e. for the strong coupling case. As has been discussed in previous papers for the investigation of strong coupling functional equations, the anharmonic oscillator is a suitable test problem ${ }^{2,4-6}$. The general idea for the solution of such functional equations is the use of an expansion of the physical functionals into series of suitably chosen base functionals and to approximate the exact infinite series by series with a finite number of terms. As has been shown in ${ }^{6}$ and ${ }^{7}$ the approximation procedure can be performed either in a symmetrical or in an unsymmetrical functional operator representation. The unsymmetrical representation has been discussed in ${ }^{7}$. In this paper we investigate the symmetrical representations. These representations require functional integrations of base functionals provided by the so-called Friedrichs-Shapiro integration procedure over functional space ${ }^{8}$. The general theory requires the proof of convergence and the explicit calculation of the approximate functionals. Only the second problem is discussed here.

1 W. Heisenberg, An Introduction to the Unified Theory of Elementary Particles, Wiley \& Sons, London 1967.

2 H. Rampacher, H. Stumpf, and F. W 13, 385 [1965].

3 H. P. Dürr and F. W

4 W. Heisenberg, Nachr. Gött. Akad. Wiss. 1953, 111.

5 H. Stumpf, F. W AGNer, and F. W AhL, Z. Naturforsch. 19 a, 1254 [1964].
For having analogy to nonlinear spinor theory we use the $p$ - $q$ representation of the anharmonic oscillator. Additionally this representation is the only one which preserves formal selfadjointness of the functional operator. It will be shown that the solution procedure runs analogous to the unsymmetrical case. For the lowest approximation numerical values are given. The value of the symmetrical representations lies in the fact, that one may hope to prove convergence. Technical details are given in the appendices. The use of Hermitean functionals for the symmetric representation has been first proposed and investigated by MAIson ${ }^{9}$. His paper gives a good complement to the more practical directed investigations given here.

\section{Spinorial Representation of the Anharmonic Oscillator}

Identifying $q$ with $\psi_{1}$ and $p$ with $\psi_{2}$ the equations of motion for the anharmonic oscillator can be written according to I

$$
\frac{\mathrm{d}}{\mathrm{d} t} \psi_{\alpha}(t)=B_{\alpha \beta} \psi_{\beta}(t)-C_{\alpha \beta} D_{\gamma \sigma} \psi_{\beta}(t) \psi_{\gamma}(t) \psi_{\sigma}(t)
$$

with the commutation relation

$$
\left[\psi_{\alpha}(t), \psi_{\beta}(t)\right]_{-}=A_{\alpha \beta} \mathbf{I}
$$

6 D. Maison and H.Stumpf, Z. Naturforsch. 21 a, 1829 [1966], in the following denoted with I.

7 W. Schuler and H. Stumpf, Z. Naturforsch. 22 a, 1842 [1967], in the following denoted with II.

${ }^{8}$ K. Friedrichs and A. Shapiro, Seminar on Integration of Functionals, New York University 1957.

9 D. Marson, Thesis, University of Munich 1967. 
where the matrices occuring in (1.1) and (1.2) are defined by

$$
\begin{array}{lll}
A:=\left(\begin{array}{rr}
0 & i \\
-i & 0
\end{array}\right), & B:=\left(\begin{array}{ll}
0 & 1 \\
0 & 0
\end{array}\right), \\
C:=\left(\begin{array}{ll}
0 & 0 \\
1 & 0
\end{array}\right), & D:=\left(\begin{array}{ll}
1 & 0 \\
0 & 0
\end{array}\right) .
\end{array}
$$

This representation is in complete analogy to the nonlinear spinor equation of elementary particle theory with Hermitean field operators ${ }^{3}$. As has been discussed in II, for the field theoretic treatment we characterize the stationary states of (1.1) by state functionals

$$
\mathfrak{I}_{\ell}(j):=\left\langle 0\left|T \exp \left\{i \int \psi_{a}(\xi) j_{a}(\xi) \mathrm{d} \xi\right\}\right| \varrho\right\rangle
$$

where $|\varrho\rangle$ are the stationary states in ordinary physical Hilbert space. For the calculation of these functionals, functional equations can be derived. Using the procedure outlined in II, app. I, we obtain the equations

$$
\begin{aligned}
\frac{\mathrm{d}}{\mathrm{d} t} \frac{\delta}{\delta j_{\alpha}(t)} \mathfrak{T}_{\varrho}(j)= & {\left[C_{a \beta} D_{\gamma \sigma} \frac{\delta}{\delta j_{\beta}(t)} \frac{\delta}{\delta j_{\gamma}(t)} \frac{\delta}{\delta_{j \sigma}(t)}\right.} \\
& \left.+B_{\alpha \beta} \frac{\delta}{\delta j_{\beta}(t)}-A_{\alpha \beta} j_{\beta}(t)\right] \mathfrak{I}_{\varrho}(j) .
\end{aligned}
$$

Additionally for the physical functionals the subsidiary condition

$$
\boldsymbol{P} \mathfrak{T}_{\varrho}(j):=\int j_{a}(t) \frac{\mathrm{d}}{\mathrm{d} t} \frac{\delta}{\delta j_{a}(t)} \mathrm{d} t \mathfrak{I}_{\varrho}(j)=-i \omega_{\varrho} \mathfrak{I}_{\varrho}(j)
$$

with $\omega_{\varrho}=\left(E_{\varrho}-E_{0}\right)$ has to be satisfied, where $E_{\varrho}$ are the energy eigenvalues for the states $|\varrho\rangle$. By (1.5) and (1.6) the physical state functionals should be characterized independently of the original problem (1.1) and (1.2) in Hilbert space, i. e. one should be able to construct the state functionals explicitly by (1.5) and (1.6). This is the main problem of the following investigation. Therefore Eqs. (1.5) and (1.6) are the starting point of our considerations.

\section{Translational Equivalent Equations}

For the purpose of numerical calculation, and as we shall see later also from general reasons it is necessary to use instead of (1.5) a suitably linear combination of the original equation. Doing so, we have to prove the equivalence of the linear combined with the original equation. Already in II such a proof of equivalence has been given for a special linear combination. Because we need in our further investigation more general linear combinations we give here a more general proof for equivalence too, showing at the same time the physical reason of equivalence. Writing the state functional (1.4) in the explicit form

$$
\begin{array}{r}
\mathfrak{I}_{\varrho}(j)=\sum_{k=1}^{\infty} \frac{i^{k}}{k !} \int\left\langle 0\left|T \psi_{a_{1}}\left(\xi_{1}\right) \ldots \psi_{a_{k}}\left(\xi_{k}\right)\right| \varrho\right\rangle \\
j_{a_{1}}\left(\xi_{1}\right) \ldots j_{a_{k}}\left(\xi_{k}\right) \mathrm{d} \xi_{1} \ldots \mathrm{d} \xi_{k}
\end{array}
$$

a simultaneous translation of all variables

$$
\xi_{i}=\xi_{i}^{\prime}+\tau
$$

gives according to properties of the time ordered products

$$
\mathfrak{T}_{Q}\left(j^{\prime}\right)=\exp \left\{i \omega_{Q} \tau\right\} \mathfrak{I}_{Q}(j)
$$

where $j^{\prime}$ has to be defined by

$$
j^{\prime}(\xi):=j(\xi+\tau) .
$$

On the other hand by a translation of all variables Eq. (1.5) remains forminvariant, i. e. the transformed equation is just the original one with $j$ replaced by $j^{\prime}$. Therefore its physical solutions do not depend on the arbitrary parameter $t$, i. e. for any arbitrary value of the parameter $t$ in (1.5) we obtain the same solutions. Therefore we are allowed to use instead of (1.5) a suitably chosen linear combination over $t$ for the calculation of $\mathfrak{T}_{\ell}(j)$ i. e. a smeared out equation. As has been shown in II the smearing out procedure can be effected even by a functional operator itself without destroying the equivalence. Then it seems, that one has a large variety of possible combinations. But these combinations are restricted by general requirements. First, as we shall see later, Eq. (1.5) is a formal Hermitean equation. For the proof of convergence of our solution procedure this property may not be destroyed. Therefore we demand

\section{The linear combination has to maintain Hermiticity.}

Secondly in the solution procedure given in II for the unsymmetrical representation, the commutativity of the equation with the translational operator $P$ is required. Because we shall apply this procedure in the symmetrical representation too, we demand 


\section{The linear combined equation has to com-} mute with $P$.

This requirement is by no means trivial, because the original Eqs. (1.5) does not commute with $P$. This indicates that (1.5) contains a larger set of solutions than the physical solutions. Therefore the smearing out procedure with the requirement 2 . can be considered as a method to select only the physical solutions of (1.5). Finally we demand

\section{The linear combined equation has to be integrable.}

This of course is meant in the light of the integration procedure developped in II. Choicing the smearing out operator $S$ to be

$$
S\left(j, \frac{\delta}{\delta j}\right):=\int \frac{\delta}{\delta j_{a}\left(t^{\prime}\right)} \delta\left(t^{\prime}-t\right) \mathrm{d} t \mathrm{~d} t^{\prime}
$$

we obtain from (1.5) by application of $S$ the equation

$$
\begin{aligned}
& \int \frac{\delta}{\delta j_{\alpha}(t)}\left[\frac{\mathrm{d}}{\mathrm{d} t} \delta_{\alpha \beta}-B_{\alpha \beta}\right] \frac{\delta}{\delta j_{\beta}(t)} \mathrm{d} t \mathfrak{I}_{Q}(j) \\
& =\int\left[C_{\alpha \beta} D_{\gamma \sigma} \frac{\delta}{\delta j_{\alpha}(t)} \frac{\delta}{\delta j_{\beta}(t)} \frac{\delta}{\delta j_{\gamma}(t)} \frac{\delta}{\delta j_{\sigma}(t)}\right. \\
& \left.\quad-\frac{1}{2} A_{\alpha \beta}\left(\frac{\delta}{\delta j_{\alpha}(t)} j_{\beta}(t)+j_{\beta}(t) \frac{\delta}{\delta j_{\alpha}(t)}\right)\right] \mathrm{d} t \mathfrak{I}_{Q}(j)
\end{aligned}
$$

where we used the fact that according to the structure of $A_{\alpha \beta}$ the source function and its differential operator can be commuted. Observing that only the commutator

$$
\left[j_{\alpha}(t), \frac{\delta}{\delta j_{\beta}\left(t^{\prime}\right)}\right]_{-}=\delta_{\alpha \beta} \delta\left(t-t^{\prime}\right)
$$

is unequal zero, while all other commutators vanish, (2.6) is a formal Hermitean equation. This means, writing (2.6) in a shorthand-notation

$$
o\left(j, \frac{\delta}{\delta j}\right) \mathfrak{T}_{\ell}(j)=0
$$

that we have for properly chosen base functionals $J_{n}$ the relation

$$
\left\langle J_{n}|O| J_{m}\right\rangle=\left\langle J_{m}|O| J_{n}\right\rangle^{*} .
$$

Therefore condition 1 . is satisfied. Defining the translational operator $P$ by

$$
\boldsymbol{P}:=\int j_{\alpha}(t) \frac{\mathrm{d}}{\mathrm{d} t} \frac{\delta}{\delta j_{\alpha}(t)} \mathrm{d} t
$$

one verifies by straight-forward calculation with the Fourier transforms of $(2.8)$ and (2.6), that $P$ commutes with (2.6). Therefore by the choice of $S$ ac- cording to (2.5) the second condition is satisfied too. Finally in section 5 and 7 we shall see that also condition 3 . is satisfied. In the following investigation we therefore use instead of (1.5) the combined Eq. (2.6).

\section{Symmetric Functional Representations}

For the actual construction of state functionals, we have to expand them into series of suitably chosen base functionals and to truncate these series. This procedure leads to matrix representations of the functional equations for the calculation of the expansion coefficients. In the preceding papers we distinguished between two possibilities of matrix representations. After substitution of the state functional expansion in the functional equation we project in one case this equation on the dual set of base functionals, in the other case on the original set. This leads to the unsymmetrical respectively symmetrical matrix representations of the functional equations. In II we treated the unsymmetrical version. In this paper we shall discuss the symmetrical version. Again we start with the Dyson expansion. Defining the spinorial Dyson base functionals by

$$
\begin{array}{r}
D_{n}\left(\begin{array}{c}
t_{1} \ldots t_{n} \\
a_{1} \ldots a_{n}
\end{array}\right)=: \frac{i^{n}}{n !} j_{a_{1}}\left(t_{1}\right) \ldots j_{a_{n}}\left(t_{n}\right) \\
\cdot \exp \left\{-\frac{1}{2} \int j_{\alpha}(\xi) F_{\alpha \beta}(\xi-\eta) j_{\beta}(\eta) \mathrm{d} \xi \mathrm{d} \eta\right\}
\end{array}
$$

where $F_{\alpha \beta}(\xi-\eta)$ is the two point function

$$
F_{\alpha \beta}(\xi-\eta):=\left\langle 0\left|T \psi_{\alpha}(\xi) \psi_{\beta}(\eta)\right| 0\right\rangle
$$

we expand $\mathfrak{I}_{Q}(j)$ into a series of Dyson base functionals

$$
\mathfrak{T}_{Q}(j)=\sum_{n=1}^{\infty} \int \varphi_{n}\left(\begin{array}{l}
\xi_{1} \ldots \xi_{n} \\
a_{1} \ldots \alpha_{n}
\end{array}\right) D_{n}\left(\begin{array}{l}
\xi_{1} \ldots \xi_{n} \\
a_{1} \ldots \alpha_{n}
\end{array}\right) \mathrm{d} \xi_{1} \ldots \mathrm{d} \xi_{n} .
$$

For the evaluation of the symmetrical matrix representation it is necessary to perform genuine functional integration between the different base functionals (3.1). This is posible only if we transform the base functionals into the standard form by a change in the source functions $j_{\alpha}(t)$. Substituting the transformation

$$
j_{\alpha}(t)=\int U_{\alpha \beta}(t-\xi) h_{\beta}(\xi) \mathrm{d} \xi
$$

in (3.1) and (3.3) where $U_{a \beta}\left(t_{1}-t_{2}\right)$ is defined by $\int U_{\beta a}(x-t) F_{\beta \gamma}(x-y) U_{\gamma \delta}\left(y-t^{\prime}\right) \mathrm{d} x \mathrm{~d} y$

$$
=\frac{1}{2} \delta_{\alpha \delta} \delta\left(t-t^{\prime}\right)
$$


we obtain the expansion

$$
\mathfrak{T}_{\varrho}(j)=\sum_{n=1}^{\infty} \int \chi_{n}\left(\begin{array}{l}
\xi_{1} \ldots \xi_{n} \\
a_{1} \ldots a_{n}
\end{array}\right) d_{n}\left(\begin{array}{l}
\xi_{1} \ldots \xi_{n} \\
a_{1} \ldots a_{n}
\end{array}\right) \mathrm{d} \xi_{1} \ldots \mathrm{d} \xi_{n}
$$

with the standard Dyson functionals of App. II

$$
\begin{array}{r}
d_{n}\left(\begin{array}{l}
\xi_{1} \ldots \xi_{n} \\
a_{1} \ldots a_{n}
\end{array} ; h\right):=\frac{i^{n}}{n !} i_{o_{1}}\left(\xi_{1}\right) \ldots h_{a_{n}}\left(\xi_{n}\right) \\
\exp \left\{-\frac{1}{4} \int h_{\alpha}^{2}(\xi) \mathrm{d} \xi\right\}
\end{array}
$$

and

$$
\begin{aligned}
\chi_{n}\left(\begin{array}{l}
\xi_{1} \ldots \xi_{n} \\
a_{1} \ldots a_{n}
\end{array}\right)= & : \int U_{a_{1} \beta_{1}}\left(\xi_{1}-t_{1}\right) \ldots U_{a_{n} \beta_{n}}\left(\xi_{n}-t_{n}\right) \\
& \times \varphi_{n}\left(\begin{array}{l}
t_{1} \ldots t_{n} \\
\beta_{1} \ldots \beta_{n}
\end{array}\right) \mathrm{d} t_{1} \ldots \mathrm{d} t_{n} .
\end{aligned}
$$

The explicit construction of $U_{a \beta}\left(t_{1}-t_{2}\right)$ can be easily achieved by transforming (3.5) into momentum space. We do not discuss this explicitly because we shall see later, that one is able to eliminate all $U_{\alpha \beta}$ in the final equations.

Having transformed the state functionals we have to do the same procedure with the functional operators. This gives with (3.4) and the resulting relation

$$
\frac{\delta}{\delta j_{\alpha}(t)}=\int U_{\beta \alpha}(t-\xi) \frac{\delta}{\delta h_{\beta}(\xi)} \mathrm{d} \xi
$$

the transformed Eq. (2.6)

$$
\begin{gathered}
\int Z_{\beta_{1} \beta_{2}}^{1}\left(t_{1} t_{2}\right) \frac{\delta}{\delta h_{\beta_{1}}\left(t_{1}\right)} \frac{\delta}{\delta h_{\beta_{2}}\left(t_{2}\right)} \mathrm{d} t_{1} \mathrm{~d} t_{2} \mathfrak{T}_{Q}(h)=\int Z_{\beta_{1} . . \beta_{4}}^{3}\left(t_{1} \ldots t_{4}\right) \prod_{i=1}^{4} \frac{\delta}{\delta h_{\beta_{i}}\left(t_{i}\right)} \mathrm{d} t_{1} \ldots \mathrm{d} t_{4} \mathfrak{T}_{Q}(h) \\
-\frac{1}{2} \int Z_{\beta_{1} \beta_{2}}^{2}\left(t_{1} t_{2}\right)\left[h_{\beta_{2}}\left(t_{2}\right) \frac{\delta}{\delta h_{\beta_{1}}\left(t_{1}\right)}+\frac{\delta}{\delta h_{\beta_{1}}\left(t_{1}\right)} h_{\beta_{2}}\left(t_{2}\right)\right] \mathrm{d} t_{1} \mathrm{~d} t_{2} \mathfrak{T}_{Q}(h)
\end{gathered}
$$

where the coefficient functions are defined by

$$
\begin{aligned}
& Z_{\beta_{1} \beta_{2}}^{1}\left(t_{1} t_{2}\right):=\int U_{\beta_{1} \alpha}^{-1}\left(t_{1}-\xi\right)\left[\frac{\mathrm{d}}{\mathrm{d} \xi} \delta_{\alpha \beta}-B_{\alpha \beta}\right] U_{\beta \beta_{1}}^{-1}\left(\xi-t_{2}\right) \mathrm{d} \xi, \\
& Z_{\beta_{1} \beta_{2}}^{2}\left(t_{1} t_{2}\right):=\int U_{\beta_{1} \alpha}^{-1}\left(t_{1}-\xi\right) A_{\alpha \beta} U_{\beta \beta_{2}}\left(\xi-t_{2}\right) \mathrm{d} \xi, \\
& Z_{\beta_{1} \ldots \beta_{4}}^{3}\left(t_{1} \ldots t_{4}\right):=\int U_{\beta_{1} \alpha}^{-1}\left(t_{1}-\xi\right) C_{\alpha \beta} U_{\beta_{2} \beta}^{-1}\left(t_{2}-\xi\right) U_{\beta_{3} \gamma}^{-1}\left(t_{3}-\xi\right) D_{\gamma \sigma} U_{\beta_{4} \sigma}^{-1}\left(t_{4}-\xi\right) \mathrm{d} \xi .
\end{aligned}
$$

The condition of stationarity (1.6) is transformed into

$$
\int U_{a \beta_{1}}\left(t-t_{1}\right) \frac{\mathrm{d}}{\mathrm{d} t} U_{\beta_{2} \alpha}^{-1}\left(t_{2}-t\right) \mathrm{d} t h_{\beta_{1}}\left(t_{1}\right) \frac{\delta}{\delta h_{\beta_{2}}\left(t_{2}\right)} \mathrm{d} t_{1} \mathrm{~d} t_{2} \mathfrak{T}_{\varrho}(h)=-i \omega_{Q} \mathfrak{T}_{Q}(h) .
$$

Further one can show, that for the transformed Eq. (3.10) the conditions 1 . and 2 . of section 2 are also satisfied. So the transition to the standard form does not disturb the general solution programme. Of course the condition 3 . is also still satisfied.

Using the abbreviations of II section 5 the symmetrical version of the theory then is defined by the matrix representation

$$
\sum_{l=1}^{\infty}\left(\sum_{i=1}^{3} Z_{i} A_{k e}^{i}\right) \chi^{l}(\varrho)=0 \quad(k=1 \ldots \infty)
$$

with

$$
\begin{aligned}
A_{k e}^{1}:= & \int d_{k}(h) \frac{\delta}{\delta h_{\beta_{1}}\left(t_{1}\right)} \frac{\delta}{\delta h_{\beta_{2}}\left(t_{2}\right)} d_{l}(h) \mathrm{d} h, \\
A_{k e}^{2}:= & \int d_{k}(h) \frac{1}{2}\left[h_{\beta_{2}}\left(t_{2}\right) \frac{\delta}{\delta h_{\beta_{1}}\left(t_{1}\right)}\right. \\
& \left.+\frac{\delta}{\delta h_{\beta_{1}}\left(t_{1}\right)} h_{\beta_{2}}\left(t_{2}\right)\right] d_{l}(h) \mathrm{d} h, \\
A_{k e}^{3}:= & \int d_{k}(h) \prod_{i=1}^{4} \frac{\delta}{\delta h_{\beta_{i}}\left(t_{i}\right)} d_{l}(h) \mathrm{d} h,
\end{aligned}
$$

where the symbol " $\mathrm{d} h$ " means the Friedrichs-Shapiro integral over $h$, discussed in App. I and App. II, the existence of which may be assumed. Applying the $h$-operators on $d_{l}(h)$ in (3.14) the result can be expressed by a linear combination of the $d_{r}(h)$ again. Therefore for the evaluation of (3.14) we only have to calculate the Friedrichs-Shapiro integral between two arbitrary standard Dyson functionals $d_{n}(h)$ and $d_{s}(h)$. According to App. I and App. II this calculation can be performed by introducing a second set of base functionals, the socalled Hermitean base functionals $J_{n}(h)$. They can be defined in terms of standard Dyson functionals by an expansion

and

$$
\begin{aligned}
& J_{n}(h)=\sum_{k=0}^{n} A_{n k} d_{k}(h) \\
& d_{n}(h)=\sum_{k=0}^{n} B_{n k} J_{k}(h)
\end{aligned}
$$

where the explicit expressions for $A_{n k}$ and $B_{n k}$ are given in App. I and App. II. There is also discussed the orthonormality of these functionals, namely

$$
\int J_{n}(h) J_{m}(h) \mathrm{d} h=\delta_{n, m} .
$$


Then by substituting the expansion (3.16) into the scalar product between $d_{n}(h)$ and $d_{s}(h)$ we obtain the result

$$
\int d_{r}(h) d_{s}(h) \mathrm{d} h=\sum_{k=0}^{\min (r, s)} B_{r k} B_{s k}:=g_{r s}
$$

also given in App. I and App. II, i. e. the standard Dyson base functionals are not orthogonal with respect to integration over Hilbert space. Therefore it seems to be advantageous to use instead of the Dyson expansion (3.6) for $\mathfrak{T}_{Q}(h)$ an expansion with Hermitean functionals. Symbolically written this reads

$$
\mathfrak{T}_{Q}(h)=\sum_{n=1}^{\infty} \eta^{n}(\varrho) J_{n}(h) \frac{1}{\sqrt{n !}}
$$

and leads to the matrix representation of the functional Eq. (3.10)

$\sum_{l=1}^{\infty}\left(\sum_{i=1}^{3} Z_{i} M_{k e}^{i}\right) \eta^{l}(\varrho) \frac{1}{\sqrt{l !}}=0 \quad(k=1 \ldots \infty)$

with

$$
\begin{aligned}
M_{k e}^{1}:= & \int J_{k}(h) \frac{\delta}{\delta h_{\beta_{1}}\left(t_{1}\right)} \frac{\delta}{\delta h_{\beta_{2}}\left(t_{2}\right)} J_{l}(h) \mathrm{d} h, \\
M_{k e}^{2}:= & \int J_{k}(h) \frac{1}{2}\left[h_{\beta_{2}}\left(t_{2}\right) \frac{\delta}{\delta h_{\beta_{1}}\left(t_{1}\right)}\right. \\
& \left.+\frac{\delta}{\delta h_{\beta_{1}}\left(t_{1}\right)} h_{\beta_{2}}\left(t_{2}\right)\right] J_{l}(h) \mathrm{d} h, \\
M_{k e}^{3}:= & \int J_{k}(h) \prod_{i=1}^{4} \frac{\delta}{\delta h_{\beta_{i}}\left(t_{i}\right)} J_{l}(h) \mathrm{d} h .
\end{aligned}
$$

Again the aplication of the h-operators on $J_{l}(h)$ in (3.21) leads finally to a linear combination of the $J_{r}(h)$ alone. Therefore the expressions (3.21) can explicitly be evaluated by the use of (3.17). This is discussed in detail in the following section and in App. III. In this section we are only interested in the basic features of these representations. First of all it can be seen, that the infinite representations, i. e. the exact Eqs. (3.13) and (3.20) are equivalent by means of a similarity transformation. $\mathrm{Ob}$ serving the invariance of $\mathfrak{T}_{Q}(h)$ against special base expansions, we obtain from (3.15) and (3.16)

$$
\chi_{l}(\varrho)=\sum_{k=1}^{\infty} A_{l k}^{\mathrm{T}} \eta_{k}(\varrho) \frac{1}{\sqrt{k !}}
$$

and

$$
\eta_{k}(\varrho)=\sum_{l=1}^{\infty} B_{k l}^{\mathrm{T}} \sqrt{k !} \chi_{l}(\varrho) .
$$

Further we have by substituting (3.16) into (3.14)

$$
A_{k e}^{i}=\sum_{r=1}^{l} \sum_{s=1}^{k} B_{k s} M_{s r}^{i} B_{r e}^{\mathrm{T}}
$$

and by inserting (3.24) into (3.13) and observing (3.21) the equivalence of (3.13) and (3.20) is verified. Additionally due to the special structure of the transformations (3.15), (3.16) the equivalence of (3.20) and (3.13) is even valid for the truncated equations. According to I and II the approximations are defined by the sequence of truncated equations

$$
\sum_{l=1}^{N}\left(\sum_{i=1}^{3} Z_{i} A_{k l}^{i}\right) \chi^{l}(N)=0 \quad(k=1 \ldots N)
$$

respectively

$$
\sum_{l=1}^{N}\left(\sum_{i=1}^{3} Z_{i} M_{k l}^{i}\right) \eta^{l}(N) \frac{1}{\sqrt{l !}}=0 \quad(k=1 \ldots N)
$$

with $N=1 \ldots \infty$, where the limit $N$ to infinity gives back the exact system (3.13) respectively (3.20). Then according to the special structure of the transformation the relations (3.24) are still exactly valid for the truncated matrices $A_{k l}^{i} \quad(k, l=1 \ldots N)$ and $M_{k l}^{i}(k, l=1 \ldots N)$ i. e. the polynomial degree of the truncation is a "good quantum number" against the transformation. Defining

$$
\eta^{j}(N)=\sum_{l=1}^{N} B_{j l}^{\mathrm{T}} \sqrt{j !} \chi^{l}(N)
$$

the truncated systems (3.25), (3.26) are also equivalent. Therefore for pure theoretical discussions we are allowed to change from one representation to the other without loss of generality. For numerical calculation one easily recognizes that the Hermitean representation is the more suitable one. This will be discussed in detail in the following section.

\section{The Hermitean Representation}

In this section we give the explicit form of the Hermitean representation (3.20). The matrix elements (3.21) are evaluated in detail in App. III. Defining

$$
Z_{\beta_{1} \beta_{2}}^{ \pm}\left(t_{1} t_{2}\right):=Z_{\beta_{1} \beta_{2}}^{1}\left(t_{1} t_{2}\right) \pm Z_{\beta_{1} \beta_{2}}^{2}\left(t_{1} t_{2}\right)
$$

we obtain the system 


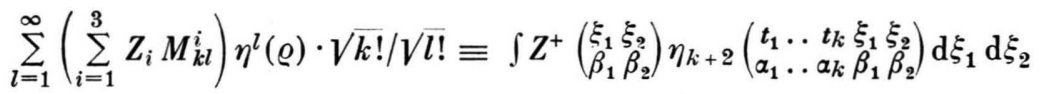

$$
\begin{aligned}
& +\sum_{\lambda_{1}=1}^{k} \int\left[Z^{+}\left(\begin{array}{cc}
t_{\lambda_{1}} & \xi \\
\alpha_{\lambda_{1}} & \beta
\end{array}\right)+Z^{-}\left(\begin{array}{ll}
\xi & t_{\lambda_{1}} \\
\beta & \alpha_{\lambda_{1}}
\end{array}\right)\right] \eta_{k}\left(\begin{array}{ccc}
t_{\lambda_{2}} & \ldots & t_{\lambda_{k}} \\
a_{\lambda_{2}} & \ldots & \alpha_{\lambda_{k}}
\end{array}\right)+\sum_{\lambda_{1} \lambda_{2}} Z^{-}\left(\begin{array}{cc}
t_{\lambda_{1}} & t_{\lambda_{2}} \\
a_{\lambda_{1}} & \alpha_{\lambda_{2}}
\end{array}\right) \eta_{k-2}\left(\begin{array}{ll}
t_{\lambda_{3}} \ldots & t_{\lambda_{k}} \\
a_{\lambda_{3}} \ldots & \alpha_{\lambda_{k}}
\end{array}\right)
\end{aligned}
$$

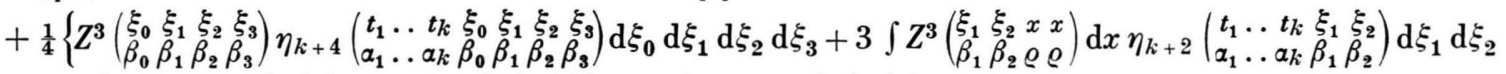

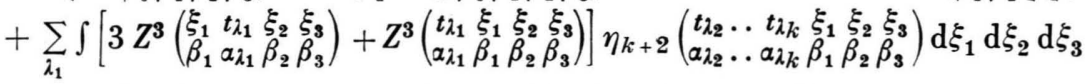

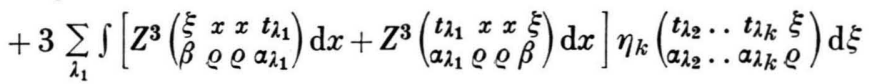

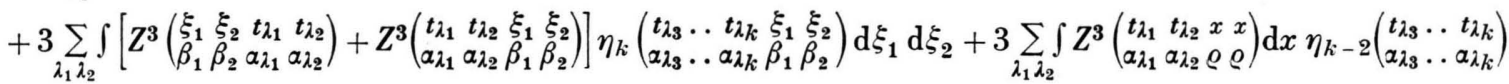

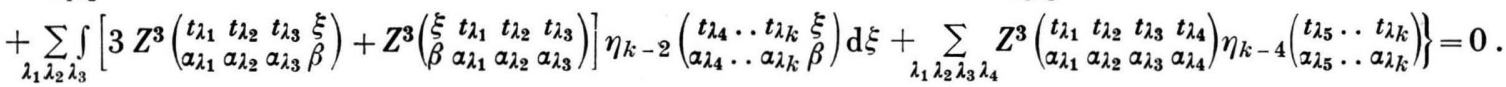

For the derivation of (4.2) use is made of the fact that we have

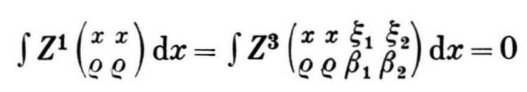

see App. III. It is interesting to note, that if we would have introduced a normal-ordering term in the fundamental Eq. (1.1) and would have used a correct functions $s\left(t-t^{\prime}\right)$ instead of a $\delta$-function in the defining expression (2.5) for the smearing out operator $S$, all terms containing $F_{11}(0)$ would cancel. This is the same behaviour like in the Dyson representation. The regularization of the interaction term by normal-ordering therefore is a universal procedure which is not confined to the unsymmetrical Dyson representation alone.

As all Feynman integrals are to be calculated in momentum space, for the effective integration of (4.2) it is still necessary to perform a Fourier transformation on (4.2). Denoting all Fourier transforms by a tilde, observing the definition of $\tilde{U}_{\alpha \beta}$ to be

$$
U_{\alpha \beta}\left(t-t^{\prime}\right)=\frac{1}{2 \pi} \int e^{-i p\left(t-t^{\prime}\right)} \tilde{U}_{\alpha \beta}(p) \mathrm{d} p
$$

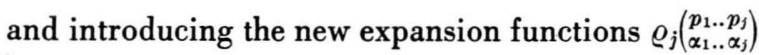
by

$$
\tilde{\eta}_{j}\left(\begin{array}{l}
p_{1} \ldots p_{j} \\
a_{1} \ldots \alpha_{j}
\end{array}\right)=\tilde{U}_{\alpha_{1} \beta_{1}}\left(p_{1}\right) \ldots \tilde{U}_{\alpha_{j} \beta_{j}}\left(p_{j}\right) \varrho_{j}\left(\begin{array}{c}
p_{1} \ldots p_{j} \\
\beta_{1} \ldots \beta_{j}
\end{array}\right)
$$

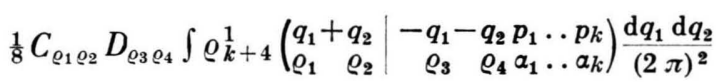

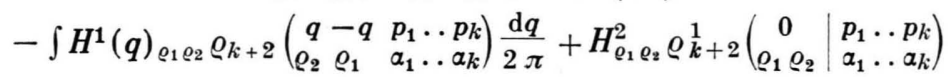

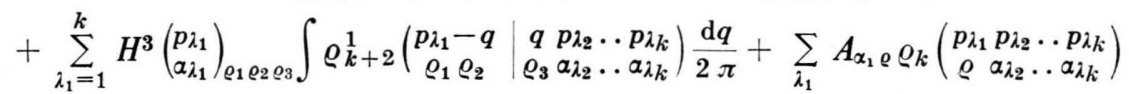

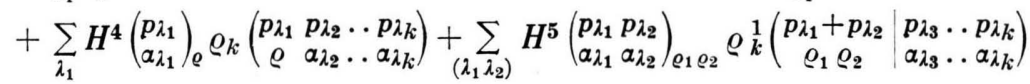

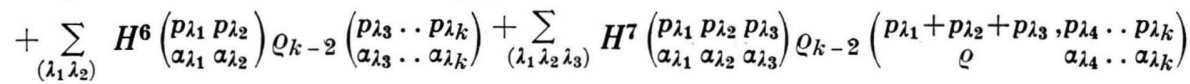

$$
\begin{aligned}
& \underset{\left(\lambda_{1} \lambda_{2} \lambda_{3} \lambda_{4}\right)}{+H^{8}}\left(\begin{array}{llll}
p_{\lambda_{1}} & p_{\lambda_{2}} & p_{\lambda_{3}} & p_{\lambda_{4}} \\
a_{\lambda_{1}} \alpha_{\lambda_{2}} & \alpha_{\lambda_{3}} & \alpha_{\lambda_{4}}
\end{array}\right) \varrho_{k-4}\left(\begin{array}{c}
p_{\lambda_{5}} \ldots p_{\lambda_{k}} \\
a_{\lambda_{5}} \ldots \alpha_{\lambda_{k}}
\end{array}\right)=0 \text {. }
\end{aligned}
$$

[Here the symbol $\left(\lambda_{1} \ldots \lambda_{k}\right)$ means summation over all combinations of $i$ elements out of $k$ elements independent of their sequence.] where the contraction operation $\varrho_{k}^{1}\left(\begin{array}{c|c}p & p_{1} \ldots p_{k-2} \\ \varrho_{1} \varrho_{1} & a_{1} \ldots \alpha_{k-2}\end{array}\right):=\frac{1}{2 \pi} \int \varrho_{k}\left(\begin{array}{ccc}p-\xi & \xi & p_{1} \ldots p_{k-2} \\ \varrho_{1} & \varrho_{2} & \alpha_{1} \ldots \alpha_{k-2}\end{array}\right) \mathrm{d} \xi$ has been used as an abbreviation. Observing further that $\tilde{U}_{a \beta}(p)$ is given by

$$
\tilde{U}_{\alpha \beta}(p) \tilde{U}_{\beta \gamma}(p)=\frac{1}{2} \tilde{F}_{\alpha \gamma}(p)
$$

the coefficient functions follow by direct calculation. We have 


$$
\begin{aligned}
& H^{1}(q)_{\varrho_{1} \varrho_{2}} \quad:=\frac{1}{2} A_{\varrho_{1} \varrho} \tilde{F}_{\varrho \varrho_{2}}^{-1}(q), \\
& H^{2}{ }_{\varrho_{1} \varrho_{2}} \quad:=\frac{1}{4}\left[a^{2} C_{\varrho_{1} \varrho_{2}}-2 B_{\varrho_{1} \varrho_{2}}\right] \text {, } \\
& H^{3}\left(\begin{array}{c}
p_{1} \\
a_{1}
\end{array}\right)_{\varrho_{1} \varrho_{2} \varrho_{3}}:=\frac{1}{4} \tilde{F}_{\alpha_{1} \beta}\left(p_{1}\right)\left[C_{\beta \varrho_{1}}+3 C_{\varrho_{1} \beta}\right] D_{\varrho_{2} \varrho_{3}}, \\
& H^{4}\left(\begin{array}{l}
p_{1} \\
a_{1}
\end{array}\right)_{\varrho} \quad:=\tilde{F}_{\alpha_{1} \beta}\left(p_{1}\right)\left\{\frac{a^{2}}{2}\left[C_{\beta_{Q}}+C_{\varrho \beta}\right]\right. \\
& \left.-\left[B_{\beta_{Q}}+B_{Q \beta}\right]\right\}+\tilde{F}_{a_{1} \beta}\left(p_{1}\right) A_{\beta \gamma} \tilde{F}_{\gamma \varrho}^{-1}\left(p_{1}\right), \\
& H^{5}\left(\begin{array}{l}
p_{1} p_{2} \\
a_{1} \alpha_{2}
\end{array}\right)_{\varrho_{1} \varrho_{2}}:=\operatorname{sym}_{1,2} \frac{3}{2} \tilde{F}_{\alpha_{1} \mu_{1}}\left(p_{1}\right) \tilde{F}_{\alpha_{2} \mu_{2}}\left(p_{2}\right) \\
& \times\left[C_{\mu_{1} \mu_{2}} D_{\varrho_{1} \varrho_{2}}+C_{\varrho_{1} \varrho_{2}} D_{\mu_{1} \mu_{2}}\right],
\end{aligned}
$$

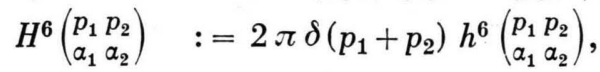

$$
\begin{aligned}
& h^{6}\left(\begin{array}{ll}
p_{1} & p_{2} \\
a_{1} & a_{2}
\end{array}\right) \quad:=\operatorname{sym}_{1,2} \tilde{F}_{a_{1} \varrho_{1}}\left(p_{1}\right) \tilde{F}_{\alpha_{2} \varrho_{2}}\left(p_{2}\right) \\
& \times 2\left[\frac{a^{2}}{2} C_{\varrho_{1} \varrho_{2}}-B_{\varrho_{1} \varrho_{2}}-i p_{1} \delta_{\varrho_{1} \varrho_{2}}-\tilde{F}_{\varrho_{1} \varrho}^{-1}\left(p_{1}\right) A_{\varrho \varrho_{2}}\right], \\
& H^{7}\left(\begin{array}{l}
p_{1} p_{2} \\
a_{1} \alpha_{2} a_{3}
\end{array}\right)_{e}:=\underset{1,2,3}{\operatorname{sym}} \tilde{F}_{a_{1} \mu_{1}}\left(p_{1}\right) \tilde{F}_{a_{2} \mu_{2}}\left(p_{2}\right) \tilde{F}_{a_{3} \mu_{3}}\left(p_{3}\right) \\
& \times\left[3 C_{\mu_{3} \varrho}+C_{\varrho \mu_{3}}\right] D_{\mu_{1} \mu_{\mathrm{s}}}, \\
& H^{8}\left(\begin{array}{l}
p_{1} \ldots p_{4} \\
a_{1} \ldots a_{4}
\end{array}\right):=2 \pi \delta\left(p_{1}+p_{2}+p_{3}+p_{4}\right) h^{8}\left(\begin{array}{l}
p_{1} \ldots p_{4} \\
a_{1} \ldots a_{4}
\end{array}\right), \\
& h^{8}\left(\begin{array}{l}
p_{1} \ldots p_{4} \\
a_{1} \ldots \alpha_{4}
\end{array}\right):=\operatorname{sym}_{1,2,3,4} \prod_{i=1}^{4} \tilde{F}_{\alpha_{i} \varrho_{i}}\left(p_{1}\right) 2 C_{\varrho_{1} \varrho_{2}} D_{\varrho_{3} \varrho_{4}} .
\end{aligned}
$$

The symbol "sym" means symmetrization over the corresponding indices, and $a^{2}=3 F_{11}(0)$. Equation (4.6) is the starting point of our integration theory for the symmetric representation given in the next section.

\section{General Solution Procedure}

The system (4.6) is a system of integral equations but not in the common sense. Irrespective of the fact that it is an infinite system, (4.6) contains "contracted" functions and a lot of "unbounded" variables, i. e. variables over which no integration has been carried out. The contracted functions are defined by (4.7). By direct inspection of (4.6) follows, that for the integration procedure of (4.6) one has no other possibility than to consider the contracted functions $\varrho_{k}^{1}$ like new unknown functions. Of course these functions are calculable. Applying the "contraction" operation (4.7) to the system (4.6) one obtains a system for the $\varrho_{k}^{1}$-functions. But this system now contains doubly contracted $\varrho$ functions etc. The necessary procedure for obtaining a closed system of integral equations can be presented in a systematic way analogous to the unsymmetrical case in II Sect. 7 . We repeat this procedure here, because we deal with spinorial functions. To perform it, we specialize to the case of state functionals for stationary states of even parity. Then only even indices $k=2,4 \ldots$ do occur in (4.6). The case of odd parity can be treated in complete analogy. Assuming now a completely symmetric set of Fourier-transformed spinorial expansion functions $\tilde{f}_{2 m}$ of a state functional $\mathfrak{F}$, we can define the following contraction operations and functions

$$
\begin{aligned}
& f_{2 m}^{k}\left(\begin{array}{c|c}
q_{1} \ldots q_{k} & \begin{array}{c}
q_{k+1} \ldots q_{2 m-k} \\
A_{1} \ldots A_{k}
\end{array} \\
a_{k+1} \ldots \alpha_{2 m-k}
\end{array}\right):=P_{k} \ldots P_{1} \tilde{f}_{2 m}\left(\begin{array}{c}
q_{1} \ldots q_{2 m} \\
a_{1} \ldots \alpha_{2 m}
\end{array}\right) \\
& :=\frac{1}{(2 \pi)^{k}} \int \tilde{f}_{2 m}\left(\begin{array}{ccccc}
q_{1}-\xi_{1} \ldots q_{k}-\xi_{k} & \xi_{1} \ldots \xi_{k} & q_{k+1} \ldots q_{2 m-k} \\
a_{1} & \ldots & \alpha_{k} & \alpha_{2 m} \ldots a_{2 m-k+1} & \alpha_{k+1} \ldots \alpha_{2 m-k}
\end{array}\right) \mathrm{d} \xi_{1} \ldots \mathrm{d} \xi_{k}
\end{aligned}
$$

with $\tilde{f}_{2 m}=: f_{2 m}^{0}$ and $A_{i}=\left(\alpha_{i}, \alpha_{2 m-i+1}\right)$. To apply the contraction operators $P_{j}$ on the system (4.6) we write it in the following form

$$
\begin{aligned}
& \frac{1}{8} C_{\varrho_{1} \varrho_{2}} D_{\varrho_{3} \varrho_{4}} \int \varrho_{k+4}^{1}\left(\begin{array}{c|cc}
q_{1}+q_{2} & -q_{1} & -q_{2} p_{1} \ldots p_{k} \\
\varrho_{1} \varrho_{2} & \varrho_{3} & \varrho_{4} \alpha_{1} \ldots \alpha_{k}
\end{array}\right) \frac{\mathrm{d} q_{1} \mathrm{~d} q_{2}}{(2 \pi)^{2}}-\int H^{1}(q)_{\varrho_{1} \varrho_{2} \varrho_{k+2}}\left(\begin{array}{ccc}
q-q & p_{1} \ldots p_{k} \\
\varrho_{2} & \varrho_{1} \alpha_{1} \ldots \alpha_{k}
\end{array}\right) \frac{\mathrm{d} q}{(2 \pi)}
\end{aligned}
$$

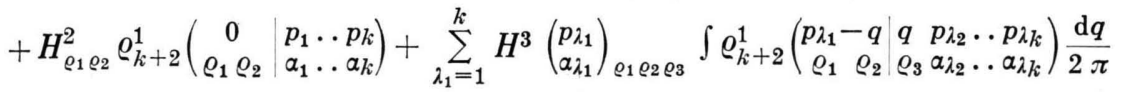

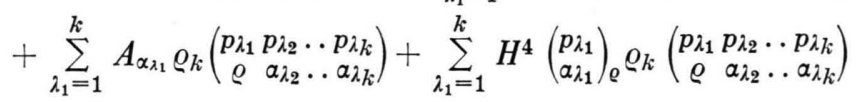

$$
\begin{aligned}
& +\sum_{\left(\lambda_{1} \lambda_{2}\right)=1}^{k} H^{5}\left(\begin{array}{c}
p_{\lambda_{1}} p_{\lambda_{2}} \\
a_{\lambda_{1}} \alpha_{\lambda_{2}}
\end{array}\right)_{\varrho_{1} \varrho_{2}} \varrho_{1}^{k}\left(\begin{array}{cc|cc}
p_{\lambda_{1}}+p_{\lambda_{2}} & p_{\lambda_{3}} \ldots p_{\lambda_{k}} \\
\varrho_{1} & \varrho_{2} & a_{\lambda_{3}} \ldots a_{\lambda_{k}}
\end{array}\right)+r_{k}\left(\begin{array}{c}
p_{1} \ldots p_{k} \\
\alpha_{1} \ldots a_{k}
\end{array}\right)=0
\end{aligned}
$$

with

$$
\begin{aligned}
& r_{k}\left(\begin{array}{c}
p_{1} \ldots p_{k} \\
a_{1} \ldots \alpha_{k}
\end{array}\right)=: \sum_{\left(\lambda_{1} \lambda_{2}\right)=1}^{k} H^{6}\left(\begin{array}{c}
p_{\lambda_{1}} p_{\lambda_{2}} \\
a_{\lambda_{1}} \alpha_{\lambda_{2}}
\end{array}\right) \varrho_{k}\left(\begin{array}{c}
p_{\lambda_{3}} \ldots p_{\lambda_{k}} \\
a_{\lambda_{3}} \ldots a_{\lambda_{k}}
\end{array}\right)
\end{aligned}
$$

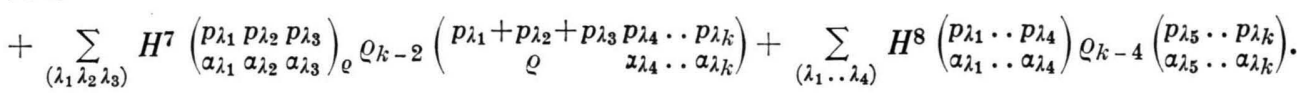


Now we apply the contraction operators $P_{k} \ldots P_{l}(k=1 \ldots m)$ to the Eqs. (5.2) for even $m$. This results in the system of equations

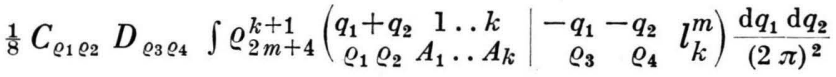

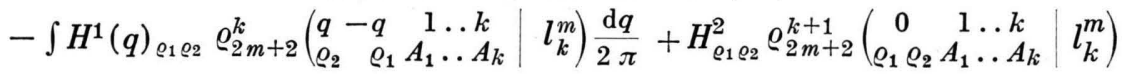

$$
\begin{aligned}
& +\sum_{\mu_{1}=1}^{k} \underset{\alpha_{\mu_{1}}, \alpha^{\prime}{ }_{\mu_{1}}}{\operatorname{sym}} \int H^{3}\left(\begin{array}{c}
\mu_{1}-\xi \\
\alpha_{\mu_{1}}
\end{array}\right)_{\varrho_{1} \varrho_{2} \varrho_{3}} \varrho_{2 m+2}^{k}\left(\begin{array}{cc|cc}
\mu_{1}-\xi_{1}-\xi_{2} & \mu_{2} \ldots \mu_{k} & \xi_{1} \xi_{2} \\
\varrho_{1} \varrho_{2} & A_{\mu_{2}} \ldots A_{\mu} & \varrho_{3} \alpha^{\prime}{ }_{\mu_{1}} & l_{k}^{m}
\end{array}\right) \frac{\mathrm{d} \xi_{1} \mathrm{~d} \xi_{2}}{(2 \pi)^{2}} \\
& +\sum_{\lambda_{1} \in l_{k}^{m}} H^{3}\left(\begin{array}{l}
\lambda_{1} \\
\alpha_{\lambda_{1}}
\end{array}\right)_{\varrho_{1} \varrho_{2} \varrho_{3}} \int \varrho_{2 m+2}^{k+1}\left(\begin{array}{cc|cc}
\lambda_{1}-q & 1 & \ldots k \\
\varrho_{1} \varrho_{2} & A_{1} & \ldots & A
\end{array} \mid \begin{array}{l}
q \\
\varrho_{3}
\end{array} l_{k, \lambda_{1}}^{m}\right) \frac{\mathrm{d} q}{2 \pi} \\
& +\sum_{\lambda_{1} \in l_{k}^{m}} A_{\alpha_{\lambda_{1}} \varrho} \varrho_{2 m}^{k}\left(\begin{array}{c}
1 \ldots k \\
A_{1} \ldots A_{k}
\end{array} \mid l_{k, \lambda_{1}}^{m} \varrho_{1}^{\lambda_{1}}\right)+\sum_{\mu_{1}=1}^{k} \underset{\alpha_{\mu_{1}, \alpha_{\mu_{1}}}}{\operatorname{sym}} A_{\alpha_{\mu_{1}} \varrho} \varrho_{2 m}^{k}\left(\begin{array}{c}
\mu_{1} \underset{\mu_{2}}{\mu_{2} \ldots \mu_{k}} \\
\varrho \alpha_{\mu_{1}} A_{\mu_{2}} \ldots A_{\mu_{k}}
\end{array} l_{k}^{m}\right)
\end{aligned}
$$

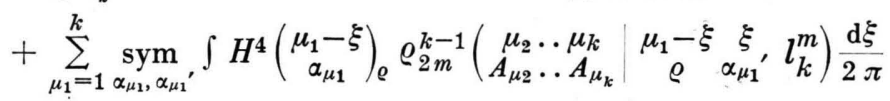

$$
\begin{aligned}
& +\sum_{\lambda_{1} \in l_{k}^{m}} H^{4}\left(\begin{array}{l}
\lambda_{1} \\
\alpha_{\lambda_{1}}
\end{array}\right)_{\varrho} \varrho_{2 m}^{k}\left(\begin{array}{c}
1 \ldots k \\
A_{1} \ldots A_{k}
\end{array} \mid \begin{array}{l}
l_{k, \lambda_{1}}^{m} \varrho_{1} \\
\lambda_{1}
\end{array}\right)+\sum_{\mu_{1}=1}^{k}{ }^{1} H^{5}\left(\begin{array}{c}
\mu_{1} \\
A_{\mu_{1}}
\end{array}\right)_{\varrho_{1} \varrho_{2}} \varrho_{2 m}^{k}\left(\begin{array}{cc}
\mu_{1} & \mu_{2} \ldots \mu_{k} \\
\varrho_{1} \varrho_{2} A_{\mu_{2}} \ldots A_{\mu_{k}}
\end{array} \mid l_{k}^{m}\right)
\end{aligned}
$$

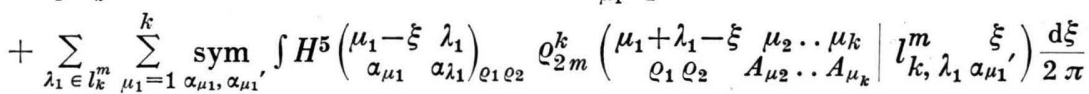

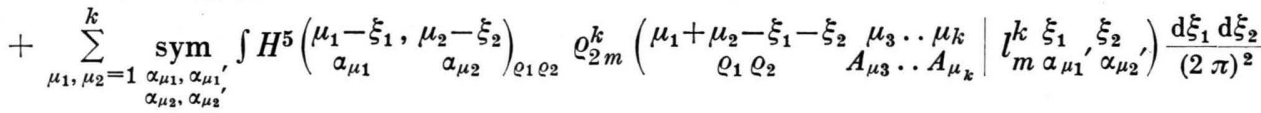

$$
\begin{aligned}
& +\sum_{\left(\lambda_{1} \lambda_{2}\right) \in l_{k}^{m}} H^{5}\left(\begin{array}{ll}
\lambda_{1} & \lambda_{2} \\
\alpha_{\lambda_{1}} & \alpha_{\lambda_{2}}
\end{array}\right)_{\varrho_{1} \varrho_{2}} \varrho_{2 m}^{k+1}\left(\begin{array}{cc}
\lambda_{1}+\lambda_{2} & 1 \ldots k \\
\varrho_{1} \varrho_{2} & A_{1} \ldots A_{k}
\end{array} \mid l_{k, \lambda_{1} \lambda_{2}}^{m}\right)+r_{m}^{k}\left(\begin{array}{c}
1 \ldots k \\
A_{1} \ldots A_{k}
\end{array} \mid l_{k}^{m}\right)=0
\end{aligned}
$$

where we denoted for brevity

$$
p_{r}=: r \quad l_{k}^{m}:=\left(\begin{array}{l}
p_{k+1} \ldots p_{2 m-k} \\
\alpha_{k+1} \ldots \alpha_{2 m-k}
\end{array}\right) .
$$

The details of its derivation are given in App. IV. It is remarkable that the system (5.4) is a closed system in the unknown functions $\varrho_{2 m}^{k}(m=1 \ldots \infty, k=0 \ldots m)$ because the Eqs. (5.4) become for $k=m$

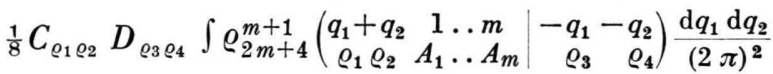

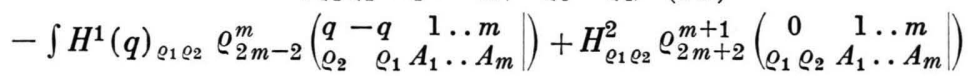

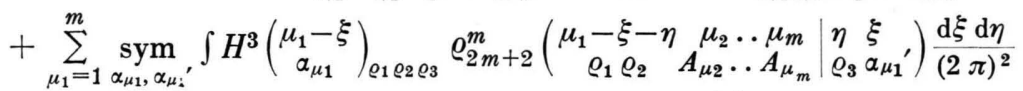

$$
\begin{aligned}
& +\sum_{\mu_{1}=1}^{m} \operatorname{sym}_{\alpha_{\mu_{1}, \alpha_{\mu_{1}}} A_{\alpha_{\mu_{1}} \varrho} \varrho_{2 m}^{m}}\left(\begin{array}{ll}
\mu_{1} & \mu_{2} \ldots \mu_{m} \\
\varrho \alpha_{\mu_{1}} & A_{\mu_{2}} \ldots A_{\mu_{m}}
\end{array}\right)+\sum_{\mu_{1}=1}^{u} \operatorname{sym}_{\alpha_{\mu_{1}, \alpha_{\mu_{1}}}} \int H^{4}\left(\begin{array}{c}
\mu_{1}-\xi \\
\alpha_{\mu_{1}}
\end{array}\right)_{\varrho} \varrho_{2 m}^{m=1}\left(\begin{array}{c}
\mu_{2} \ldots \mu_{m} \\
A_{\mu_{2}} \ldots A_{\mu_{m}}
\end{array} \mid \begin{array}{cc}
\mu_{1}-\xi & \xi \\
\varrho & \alpha_{\mu_{1}}{ }^{\prime}
\end{array}\right) \frac{\mathrm{d} \xi}{2 \pi}
\end{aligned}
$$

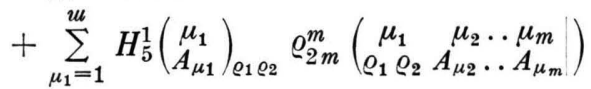

$$
\begin{aligned}
& +\sum_{\mu_{1}, \mu_{2}=1}^{u} \operatorname{sym}_{\substack{\alpha_{\mu_{1}}, \alpha_{\mu_{1}^{\prime}}, \alpha_{\alpha_{3},}, \alpha_{\mu_{2}}}} \int H^{5}\left(\begin{array}{cc|c}
\mu_{1}-\xi_{1}, \mu_{2}-\xi_{2} \\
\alpha_{\mu_{1}} & \alpha_{\mu_{2}}
\end{array}\right)_{\varrho_{1} \varrho_{2}} \varrho_{2 m}^{m-1}\left(\begin{array}{ccc}
\mu_{1}+\mu_{2}-\xi_{1}-\xi_{2} & \mu_{3} \ldots \mu_{m} \\
\varrho_{1} \varrho_{2} & A_{\mu_{3}} \ldots A_{\mu_{m}} & \xi_{1}, \xi_{2} \\
\alpha_{\mu_{1}} & \xi_{\mu_{2}}
\end{array}\right) \frac{\mathrm{d} \xi_{1} \mathrm{~d} \xi_{2}}{(2 \pi)^{2}} \\
& \left.+r_{2 m}^{m}\left(\begin{array}{c}
1 \ldots m \\
A_{1} \ldots A_{m}
\end{array}\right)\right)=0 \text {. }
\end{aligned}
$$

The last term $r_{2 m}^{m}$ reads in a symbolic notation, the precise details of which being given in App. IV

$$
r_{2 m}^{m}\left(\begin{array}{c}
1 \ldots m \\
A_{1} \ldots A_{m}
\end{array}\right)=\sum_{\mu=m-1}^{m-2} \sum_{\nu=\mu}^{\mu+2} H_{m \mu}^{m v} \varrho_{2 \mu}^{v} .
$$

Therefore the system for thte contraction functions $\varrho_{2 m}^{k}(k=0 \ldots m)$ terminates with $\varrho_{2 m}^{m}$ and no higher contraction term occurs. So (5.4) can be used instead of (5.2) 
For the solution of the infinite system we now use approximate functionals

$$
\begin{aligned}
\widetilde{\mathfrak{T}}_{Q}{ }^{N}(h): & =\sum_{m=1}^{N} \eta_{2 m}(N) J_{2 m}(h) \frac{1}{\sqrt{(2 m) !}} \\
& =\sum_{m=1}^{N} \tilde{U}^{2 m} \varrho_{2 m}(N) J_{2 m}(h) \frac{1}{\sqrt{(2 m) !}} .
\end{aligned}
$$

Formally the use of (5.8) can be defined by putting $\varrho_{2 \alpha}=0$ for $\alpha>N$ and then calculating $\varrho_{2} \ldots \varrho_{2 N}$ from the first $N$ equations of (5.2). By our contraction procedure we transformed (5.2) into (5.4) and perform calculations with this system. From (5.1) follows in this case that $\varrho_{2 \alpha}^{k}=0$ for $\alpha>N$ and $k=0 \ldots \alpha$. Therefore the truncation procedure for Eq. (5.4) is defined by putting $\varrho_{2 \alpha}^{k}=0$ for $\alpha>0$, $k=0 \ldots \alpha$ and then calculating $\varrho_{2 \alpha}^{k}$ for $\alpha=1 \ldots . N$ and $k=0 \ldots \alpha$ from the corresponding Eqs. of (5.4). Multiplying (5.4) by $\left(\sum_{\lambda_{1}=1}^{2 m} A_{\alpha_{\lambda_{i}} e}\right)^{-1}$ the sixth term in (5.4) with $\varrho_{2 m}^{k}$ becomes a diagonal term and we can write (5.4) in the symbolic form

$$
\varrho_{2 m}^{k}=\sum_{n=m-2}^{m+2} \sum_{l=k-4}^{k+1} H_{m n}^{k l} \varrho_{2 n}^{l}\left(\begin{array}{c}
m=1 \ldots \infty \\
k=0 \ldots m
\end{array}\right) \text {. }
$$

We obtain for the calculation of (5.8) the truncated system

$$
\varrho_{2 m}^{k}(N)=\sum_{n=m-2}^{m+2} \sum_{\substack{\leq=k-4 \\
\leq N}}^{k+1} H_{m n}^{k l} \varrho_{2 n}^{l}(N)\left(\begin{array}{c}
m=1 \ldots N \\
k=0 \ldots m
\end{array}\right) .
$$

Then we have to integrate (5.10) explicitly. This will be done for a simple example in detail in section 7 . Here we only want to sketch the general method. To do this it is not necessary to write down all indices explicitly. We rather use a shorthand notation. We define a subset of functions $\varrho_{2 m}^{k}$ by

$$
\boldsymbol{R}(\alpha, \beta):=\varrho_{2 m}^{k}\left(\begin{array}{c}
m=a \ldots \beta \\
k=0 \ldots m
\end{array}\right)
$$

and for any operator $\boldsymbol{O}_{m n}^{k l}$ we define its projection on this subset by

$$
\boldsymbol{O}(\alpha, \beta):=O_{m n}^{k l}\left(\begin{array}{c}
m, n=\alpha \ldots \beta \\
k, l=0 \ldots m, n
\end{array}\right) .
$$

Now we start with the lowest possible equation of (5.10) for $\varrho_{2}^{0}$ which reads

$$
\varrho_{2}^{0}(N)=H_{11}^{00} \varrho_{2}^{0}(N)+H_{11}^{01} \varrho_{2}^{1}(N)+\sum_{k l} H_{1 k}^{0 l} \varrho_{2 k}^{l}(N) .
$$

Then the remaining equations of (5.10) read in the notation of (5.11) and (5.12)

$$
\boldsymbol{R}_{N}(2, N)=\boldsymbol{H}(2, N) \boldsymbol{R}_{N}(2, N)+\boldsymbol{F}(2, N)
$$

where $\boldsymbol{F}$ contains all terms with $\varrho_{2}^{0}$ and $\varrho_{2}^{1}$ i. e.

$$
\mathbf{F}(2, N)=: \sum_{a=0}^{1} H_{m 1}^{k \alpha} \varrho_{2}^{a}(N)\left(\begin{array}{c}
m=2 \ldots N \\
k=0 \ldots m
\end{array}\right) .
$$

Then we construct the Green function for (5.14) namely

$$
\boldsymbol{G}(2, N):=[\mathbf{1}(2, N)-\boldsymbol{H}(2, N)]^{-1}
$$

and apply it to (5.14) obtaining so

$$
\boldsymbol{R}_{N}(2, N)=\mathbf{G}(2, N) F(2, N) .
$$

Explicitly (5.17) reads

$$
\varrho_{2 r}^{h}(N)=\sum_{n, l=1}^{N} G_{r n}^{h l}(N) \sum_{a=0}^{1} H_{n 1}^{l a} \varrho_{2}^{a}(N)
$$

This inserted into (5.13) leads to an equation of the structure

$$
\varrho_{2}^{0}(N)=S_{1} \varrho_{2}^{0}+S_{2} \varrho_{2}^{1}
$$

or by inversion

$$
\varrho_{2}^{0}(N)=\left(1-S_{1}\right)^{-1} S_{2} \varrho_{2}^{1}(N)=: Q(N) \varrho_{2}^{1}(N) .
$$

When we apply the contraction operation on $(5.20)$ we have

$$
\left[1-Q^{1}(N)\right] \varrho_{2}^{1}(N)=0
$$

and after introduction of center of gravity coordinates by

$$
\varrho_{2}^{1}(q)=\int \varrho_{2}^{0}(q-\xi, \xi) \mathrm{d} \xi=c_{0} \delta(q-\omega)
$$

we obtain an eigenvalue equation for the calculation of the approximate eigenvalue $\omega_{N}$ corresponding to the approximate functional $(5.8)$;

$$
Q^{1}\left(N ; \omega_{N}\right)=1
$$

of course the main calculational problem is the construction of $\mathbf{G}(2, N)$.

\section{The Condition of Stationarity}

Stationary functionals are characterized by the condition (1.6) respectively (3.12). Therefore we have to demonstrate that the solution procedure sketched in the preceding section and leading to an eigenvalue equation does satisfy (3.12) i. e. that the eigenvalue $\omega_{N}$ calculated according to section 5 is the required one by the eigenvalue condition (3.12). To do this we first represent (3.12) by Hermite functionals. Using the Hermite expansion for the state functional (3.19), Eq. (3.12) can be 
written in our symbolic notation

$$
\sum_{l=1}^{\infty} P_{k l} \eta^{l}(\varrho) \frac{\sqrt{k !}}{\sqrt{l !}}=-i \omega_{\varrho} \eta^{k}(\varrho)
$$

with

$$
\begin{aligned}
P_{k l}:=\int J_{k}(h) & U_{\alpha \beta_{1}}\left(t-t_{1}\right) \frac{\mathrm{d}}{\mathrm{d} t} U_{\beta_{2} \alpha}\left(t_{2}-t\right) h_{\beta_{1}}\left(t_{1}\right) \\
& \frac{\delta}{\delta h_{\beta_{2}}\left(t_{2}\right)} \mathrm{d} t \mathrm{~d} t_{1} \mathrm{~d} t_{1} J_{l}(h) \mathrm{d} h
\end{aligned}
$$

Explicit evaluation gives according to App. III.

$P_{k l}=$

$$
\delta_{k l} \sum_{\lambda_{1}=1}^{k} S\left(t_{\lambda_{1}}-t_{1}^{\prime}\right) \delta_{\alpha_{\lambda_{1}}, \alpha_{1}^{\prime}} \prod_{i=2}^{k} \delta_{\alpha_{\lambda_{i}}, \alpha_{i}} \delta\left(t_{\lambda_{i}}-t_{i}^{\prime}\right)
$$

i. e. $P_{k l}$ is a diagonal operator. Transforming (6.1) into Fourier space then it leads to the equation

$$
\left[\sum_{i=1}^{n} p_{i}\right] \tilde{\eta}_{n}\left(\begin{array}{l}
p_{1} \ldots p_{n} \\
a_{1} \ldots a_{n}
\end{array}\right)=\omega_{Q} \tilde{\eta}_{n}\left(\begin{array}{l}
p_{1} \ldots p_{n} \\
a_{1} \ldots a_{n}
\end{array}\right) .
$$

Transition to the $\varrho$-functions according to (4.5) finally results in the condition

$$
\left[\sum_{i=1}^{n} p_{i}\right] \varrho_{n}\left(\begin{array}{l}
p_{1} \ldots p_{n} \\
a_{1} \ldots a_{n}
\end{array}\right)=\omega_{\varrho} \varrho_{n}\left(\begin{array}{l}
p_{1} \ldots p_{n} \\
a_{1} \ldots a_{n}
\end{array}\right) .
$$

It is the same condition like in the unsymmetrical case. Specializing on states of even parity, we may apply the contraction operation of section 5 on (6.5) and obtain in this way the conditions

$\left[\sum_{i=1}^{2 m-k} p_{i}-\omega_{2}\right] \varrho_{2 m}^{k}\left(\begin{array}{c|c}p_{1} \ldots p_{k} & p_{k+1} \ldots p_{2 m-k} \\ A_{1} \ldots A_{k} & a_{k+1} \ldots a_{2 m-k}\end{array}\right)=0$.

So far Eqs. (6.1) to (6.6) are valid for the exact stationary state functionals. Assuming now an approximate functional (5.8), not only the dynamical Eqs. (5.5) have to be truncated, but also the Eq. (6.1) respectively (6.6). This gives for (6.1)

$$
\begin{aligned}
\sum_{l=1}^{N} P_{k l} \eta^{l}(N) \frac{\sqrt{k !}}{\sqrt{l !}} & =\sum_{l=1}^{\infty} P_{k l} \eta^{l}(N) \frac{\sqrt{k !}}{\sqrt{l !}} \\
& =-i \omega_{\varrho} \eta_{k}(N)
\end{aligned}
$$

due to the diagonal structure of $P_{k l}$. Therefore (3.12) has to be exactly valid for the approximate functionals too. Of course then Eqs. (6.6) have to be satisfied by the $\varrho_{2 m}^{k}(N)$ also. Now the problem can be formulated as follows: According to section 5 only $\varrho_{2}^{1}$ and $\varrho_{2}^{0}$ are properly chosen to satisfy (6.6). If one calculates the higher $\varrho$-functions by the outlined procedure, do then all $\varrho_{2 m}^{k}(N)$ satisfy (6.6) or not?
According to II, section 8 all $\varrho_{2 m}^{k}(N)$ satisfy (6.6) if $P_{k l}$ commutes with the operator of the dynamical equation. This is true according to condition 2 . of section 2. i. e. the dynamical operator is just selected from the viewpoint of commutativity. Therefore our calculation programm of section 5 automatically leads to properly constructed functionals of stationary states. For details we refer to II.

\section{Integration of an Approximate System}

The principles for the explicit integration of the truncated systems (5.6) are just the same as we have outlined in paper II section 9, where we demonstrated the method of integration for the simplest cases of the $\varphi_{2}$ and $\varphi_{2}-\varphi_{4}$-systems. The main feature is, that in the equation systems occur linear combinations of integral operators which only act partially on the whole $\varrho$-functions. And one has to construct the partial resolvents of these integral operator components. Therefrom one gets the total resolvent by an linear symmetric combination of the structure:

$$
R=1+\sum_{i} R_{i}
$$

the $R_{i}$ being the partial resolvents of the partial integral operators $K_{1}$.

But now, in the spinorial case, the whole procedure is much more complicated than in the scalar one because each partial resolvent is a matrix resolvent. Moreover, the equations have more terms and additionally one has to construct inverse matrices by the same method. Thus we don't demonstrate the method of integration in detail for some appropriate system because it would not yield new aspects.

However, we will calculate some eigenvalue for the simplest case of the $\varrho_{1}$-equation. This reads according to (4.6) if we put $\varrho_{3} \equiv 0$

$$
A_{\alpha_{1} \lambda} \varrho_{1}\left(\begin{array}{c}
p_{1} \\
\lambda
\end{array}\right)+H^{4}\left(\begin{array}{c}
p_{1} \\
a_{1}
\end{array}\right) \varrho_{1}\left(\begin{array}{c}
p_{1} \\
\lambda
\end{array}\right)=0 .
$$

Putting now

$$
\varrho_{1}\left(\begin{array}{l}
p_{1} \\
a_{1}
\end{array}\right)=\varrho_{1}^{1}\left(a_{1}\right) \delta\left(p_{1}-\omega\right)=: \varrho_{\lambda_{1}} \delta\left(p_{1}-\omega\right)
$$

as a consequence of the translational condition (6.5), we have after integration over $p_{1}$

$$
\left[A_{\alpha \beta}+H_{\alpha \beta}^{4}(\omega)\right] \varrho_{\beta}=0 .
$$

In this simple case our problem turns out to be only an algebraic one, and we get from (7.3) the eigen- 
value equation

$$
\operatorname{det}\left|A_{\alpha \beta}+H_{\alpha \beta}^{4}(\omega)\right|=0 .
$$

Regarding the definition (4.9) of $H^{4}\left(\begin{array}{c}\omega \\ \alpha\end{array}\right)_{\beta}$, we multiply (7.3) from the left by $\tilde{F}_{\alpha \beta}^{-1}(\omega)$ and get the modified eigenvalue equation

$$
\operatorname{det}\left|F^{-1} A+A F^{-1}+\frac{1}{2} a^{2}\left[C+C^{\mathrm{T}}\right]-\left[B+B^{\mathrm{T}}\right]\right|=0 .
$$

This may be written

$$
\operatorname{det}\left|F^{-1} A+A F^{-1}+c_{0} S\right|=0
$$

with

$$
c_{0}:=\frac{1}{2} a^{2}-1=\frac{3}{2} F_{11}(0)-1
$$

and

$$
S:=\left(\begin{array}{ll}
0 & 1 \\
1 & 0
\end{array}\right)
$$

having substituted the definition (1.3) of the matrices $B$ and $C$ in (7.5). As in paper II, App. V we then approximate the two-point function

$$
F_{\alpha \beta}\left(t_{1}-t_{2}\right)=\left\langle 0\left|T \psi_{\alpha}\left(t_{1}\right) \psi_{\beta}\left(t_{2}\right)\right| 0\right\rangle
$$

by its first intermediate state and use the approximate $f$-sum rule

$$
|\langle 0|q(0)| 1\rangle|^{2} 2 \omega_{10}=1
$$

as well as the relation

$$
\langle 1|p(t)| 0\rangle=i \omega_{10}\langle 1|q(t)| 0\rangle .
$$

Then we have for the Fourier-transformed $\tilde{F}_{i k}(p)_{\text {app. }}$ :

and its inverse

$$
\tilde{F}_{i k}(p)_{\text {app. }}=\frac{1}{\left[p^{2}-\omega_{10}^{2}\right]}\left(\begin{array}{cc}
i & -p \\
p & i \omega_{10}^{2}
\end{array}\right)
$$

$$
\tilde{F}_{i k}(p)^{-1} \text { app. }\left(\begin{array}{cc}
i \omega_{10}^{2} & p \\
-p & i
\end{array}\right)
$$

as well as for $c_{0}$

$$
c_{0}=\frac{3}{2} F_{11}(0)-1=\frac{3}{4 \omega_{10}}-1 .
$$

With these approximate functions and the definition (1.3) of $A$ we can immediately evaluate Eq. (7.6) resulting in

$$
\begin{array}{r}
\operatorname{det}\left|F^{-1} A+A F^{-1}+c_{0} S\right| \\
\equiv-4 \omega^{2}+\left(\omega_{10}^{2}+1\right)^{2}-c_{0}^{2}=0 .
\end{array}
$$

Looking for a selfconsistent solution we have to put $\omega_{10} \equiv \omega$ and get

$$
\left(\omega_{10}^{2}-1\right)^{2}=c_{0}^{2}
$$

and therefore

$$
\omega_{10}=\sqrt[3]{\frac{3}{4}} \cong 0.9085
$$

\footnotetext{
10 W. Schuler, Thesis, University of Tübingen 1967.
}

whereas the exact value is given by $\omega_{10}=1.0871$. It is remarkable that one gets the same value as in the Hermitean formulation of the $q$-representation ${ }^{9}$. Moreover we see that we get in the Hermitean representation in the lowest approximation an eigenvalue which is lower than the exact one, whereas the unsymmetrical version results in a higher one. There we got

$$
\omega_{10}=\sqrt[3]{\frac{3}{2}} \cong 1.1447 .
$$

A further comparison of the numerical values for the unsymmetrical and the Hermitean formulation of the $q$-representation will be given in a later paper ${ }^{10}$.

\section{Acknowledgements}

We thank very much Prof. Dr. W. Heisenberg and Dr. H. P. DürR of the Max-Planck-Institut für Physik und Astrophysik, Munich, for their kind and critical reading of the manuscript. We are also very indebted to Dipl.-Phys. R. WEBER for controlling our calculations.

\section{Appendix I}

In this part we discuss the scalar Hermite- and Dyson functionals and their integration. Hermite functionals have been introduced by Friedrichs and their integration by Friedrichs and Shapiro. Most of the formulae compiled here are given in ${ }^{8}$, as well as in ${ }^{11}$.

First we give the definition of Hermitean polynomials. Suppose to have a polynomial

$$
P_{n}(j):=\int p_{n}\left(x_{1} \ldots x_{n}\right) j\left(x_{1}\right) \ldots j\left(x_{n}\right) \mathrm{d} x_{1} \ldots \mathrm{d} x_{n}
$$

where $p_{n}\left(x_{1} \ldots x_{2}\right)$ is a real symmetric function in all variables, and sufficient regular that the integral exists. The functions $j(x)$ have to be elements of the real Hilbert space $L^{2}(-\infty,+\infty)$ i. e.

$$
\int_{-\infty}^{+\infty}|j(x)|^{2} \mathrm{~d} x<\infty
$$

Then the Hermitean Polynomial corresponding to (I.1) is defined by

$$
\begin{aligned}
H P_{n}(j): & =P_{n}\left(j-\frac{\delta}{\delta j}\right) \cdot 1 \\
& =(-)^{n} e^{\frac{1}{2} \int j^{2}(x) \mathrm{d} x} P_{n}\left(\frac{\delta}{\delta j}\right) e^{-\frac{1}{2} \int j^{2}(x) \mathrm{d} x} .
\end{aligned}
$$

11 K. Symanzik, Z. Naturforsch. 9 a, 809 [1954], appendix. 
A useful description of (I.2) is also given by

$$
\begin{aligned}
\boldsymbol{P}_{n}\left(j-\frac{\delta}{\delta j}\right) \cdot \boldsymbol{1}= & \exp \left\{-\frac{1}{2} \int \frac{\delta^{2}}{\delta j(x)^{2}} \mathrm{~d} x\right\} \boldsymbol{P}_{n}(j) \\
& =\sum_{k=0}^{[n / 2]} a_{k}\left[\int \frac{\delta^{2}}{\delta j(x)^{2}} \mathrm{~d} x\right]^{k} \boldsymbol{P}_{n}(j)
\end{aligned}
$$

with

$$
\boldsymbol{a}_{k}:=(-)^{k} /\left(k ! 2^{k}\right) \text {. }
$$

Like in ordinary function space the Hermitean polynomials are not integrable. This is only possible by introducing a weightfactor. This then leads to the definition of the Hermitean functionals

$$
J_{n}(j):=\frac{1}{\sqrt{n !}} H P_{n}(j) e^{-\frac{1}{4} \int j^{2}(x) \mathrm{d} x} .
$$

They are square integrable and their integral over functional space leads to

$$
\int J_{m}(j) J_{n}(j) \mathrm{d} j=\delta_{n, m} \int p_{n}^{2}\left(x_{1} \ldots x_{n}\right) \mathrm{d} x_{1} \ldots \mathrm{d} x_{n}
$$

To obtain from (I.6) conventional orthogonality relations as one may expect for complete orthogonal expansion systems, the functions $p_{n}\left(x_{1} \ldots x_{n}\right)$ have to be specified further. They have to be constructed as complete orthogonal sets in ordinary function space.

A special representation which does not lead to contradictions is given by

$$
p_{n}\left(t_{1} \ldots t_{n} ; x_{1} \ldots x_{n}\right):=\frac{1}{n !} P \sum_{\lambda_{1} . . \lambda_{n}=1}^{n} \prod_{\alpha=1}^{n} \delta\left(t_{\lambda \alpha}-x_{\alpha}\right)
$$

Then the numbers $t_{1} \ldots t_{n}$ which are continuously variable play the role of index numbers like the wavevectors $K$ for plane wave functions. The possibility of choosing a base system like (I.7) is in so far important and of special interest, as it allows immediately a Lorentz-invariant generalization, which finally is the aim of the entire formalism developped here. Naturally the Fourier transform of (I.7) is also a possible expansion system, but this does not lead to any new results.

By (I.7) and (I.1) the special set of Polynomials

$$
P_{n}\left(t_{1} \ldots t_{n} ; j\right):=j\left(t_{1}\right) \ldots j\left(t_{n}\right)
$$

is defined, and the corresponding set of Hermite functionals is given by

$$
J_{n}\left(t_{1} \ldots t_{n} ; j\right):=\frac{1}{\sqrt{n !}} H j\left(t_{1}\right) \ldots j\left(t_{n}\right) e^{-\frac{1}{4} \int j^{2}(x) \mathrm{d} x} .
$$

They satisfy the orthogonality relations

$$
\int J_{n}\left(t_{1} \ldots t_{n} ; j\right) J_{m}\left(t_{1}^{\prime} \ldots t_{m}^{\prime} ; j\right) \mathrm{d} j=\delta_{n m} \frac{1}{m !} P \sum_{\lambda_{1} . . \lambda_{m}=1}^{u u} \prod_{i=1}^{m} \delta\left(t_{\lambda_{i}}-t_{i}^{\prime}\right)
$$

For the explicit evaluation of matrix elements the following recursion formulas are necessary

$$
\begin{gathered}
j(t) J_{n}\left(t_{1} \ldots t_{n} ; j\right)=\sqrt{n+1} J_{n+1}\left(t_{1} \ldots t_{n} t ; j\right)+\frac{1}{\sqrt{n}} \sum_{l=1}^{n} \delta\left(t-t_{l}\right) J_{n-1}\left(t_{1}-t_{l-1} t_{l+1} \ldots t_{n} ; j\right) \\
\frac{\delta}{\delta j(t)} J_{n}\left(t_{1} \ldots t_{n} ; j\right)=\frac{1}{\sqrt{n}} \frac{1}{2} \sum_{l=1}^{n} \delta\left(t-t_{l}\right) J_{n-1}\left(t_{1} \ldots t_{l-1} t_{l+1} \ldots t_{n} ; j\right)-\frac{1}{2} \sqrt{n+1} J_{n+1}\left(t_{1} \ldots t_{n} t ; j\right) .
\end{gathered}
$$

They are proved by similar techniques like those used for ordinary Hermite functions.

Now we turn to the Dyson functionals. The normalized Dyson functionals are defined by

$$
d_{n}\left(t_{1} \ldots t_{n} ; j\right):=\frac{1}{n !} P_{n}\left(t_{1} \ldots t_{n} ; j\right) e^{-\frac{1}{4} \int j^{2}(x) \mathrm{d} x}
$$

Observing the definition (I.3) we obtain the connection between the Dyson- and Hermite functionals by

$$
\begin{aligned}
& J_{n}\left(t_{1} \ldots t_{n} ; j\right)=\frac{1}{n !} P \sum_{\lambda_{1} . . \lambda_{n}=1}^{n} \sum_{k=0}^{[n / 2]} a_{n k} \prod_{i=1}^{k} \delta\left(t_{\lambda_{2 i}}-t_{\lambda_{2 i-1}}\right) d_{n-2 k}\left(t_{\lambda_{2 k+1}} \ldots t_{n} ; j\right), \\
& d_{n}\left(t_{1} \ldots t_{n} ; j\right)=\frac{1}{n !} P \sum_{\lambda_{1} \ldots \lambda_{n}=1} \sum_{k=0}^{n} b_{n k} \prod_{i=1}^{n} \delta\left(t_{\lambda_{2 i}}-t_{\lambda_{2 i-1}}\right) J_{n-2 k}\left(t_{\lambda_{2 k+1}} \ldots t_{n} ; j\right)
\end{aligned}
$$

with

$$
a_{n k}=: \frac{(-)^{k} \sqrt{n !}}{k ! 2^{k}}, \quad b_{n k}=: \frac{1}{2^{k} \sqrt{n-2 k) ! \cdot k !}} .
$$


The inversion formula (I.15) can be obtained by reversing Schmidt's orthogonalisation procedure. The connection between both sets can be written as a similarity transformation given by

$$
\begin{aligned}
& J_{n}\left(t_{1} \ldots t_{n} ; j\right)=\sum_{r=0}^{n} \int A_{n r}\left(t_{1} \ldots t_{n} ; \xi_{1} \ldots \xi_{r}\right) d_{r}\left(\xi_{1} \ldots \xi_{r}\right) \mathrm{d} \xi_{1} \ldots \mathrm{d} \xi_{r} \\
& d_{n}\left(t_{1} \ldots t_{n} ; j\right)=\sum_{r=0}^{n} \int B_{n r}\left(t_{1} \ldots t_{n} ; \xi_{1} \ldots \xi_{r}\right) J_{r}\left(\xi_{1} \ldots \xi_{r}\right) \mathrm{d} \xi_{1} \ldots \mathrm{d} \xi_{r}
\end{aligned}
$$

with

$$
\begin{aligned}
& A_{n r}\left(t_{1} \ldots t_{r} ; \xi_{1} \ldots \xi_{r}\right):=a_{n,(n-r) / 2} \frac{1}{n !} P \sum_{\lambda_{1} \ldots \lambda_{n}=1}^{n} \prod_{i=\frac{1}{2} r+1}^{\frac{1}{2} n} \delta\left(t_{\lambda_{2 i}}-t_{\lambda_{2 i-1}}\right) \prod_{\alpha=1}^{r} \delta\left(\xi_{a}-t_{\lambda x}\right) \\
& B_{n r}\left(t_{1} \ldots t_{r} ; \xi_{1} \ldots \xi_{r}\right):=b_{n,(n-r) / 2} \frac{1}{n !} P \sum_{\lambda_{1} \ldots \lambda_{n}=1}^{n} \prod_{i=\frac{1}{2} r+1}^{\frac{1}{2} n} \delta\left(t_{\lambda_{2 i}}-t_{\lambda_{2 i-1}}\right) \prod_{\alpha=1}^{r} \delta\left(\xi_{\alpha}-t_{\lambda x}\right) .
\end{aligned}
$$

Having for a functional $\mathfrak{I}_{\varrho}(j)$ the expansion

$$
\mathfrak{I}_{Q}(j)=\sum_{n=1}^{\infty} \chi_{n} d_{n}(j)=\sum_{n=1}^{\infty} \eta_{n} J_{n}(j) \frac{1}{\sqrt{n !}}
$$

the expansion coefficients are connected by the transposed matrices

$$
\begin{aligned}
& \chi_{r}\left(\xi_{1} \ldots \xi_{r}\right)=\sum_{k=1}^{\infty} \int A_{k r}\left(t_{1} \ldots t_{k} ; \xi_{1} \ldots \xi_{r}\right) \eta_{k}\left(t_{1} \ldots t_{k}\right) \mathrm{d} t_{1} \ldots \mathrm{d} t_{k} \frac{1}{\sqrt{k !},} \\
& \eta_{r}\left(\xi_{1} \ldots \xi_{r}\right)=\sum_{k=1}^{\infty} \int B_{k r}\left(t_{1} \ldots t_{k} ; \xi_{1} \ldots \xi_{r}\right) \chi_{k}\left(t_{1} \ldots t_{k}\right) \mathrm{d} t_{1} \ldots \mathrm{d} t_{k} \cdot \sqrt{r !} .
\end{aligned}
$$

This is an essentially nonlocal connection. Especially the physically meaningful one-time limit of $\chi_{r}\left(\xi_{1} \ldots \xi_{r}\right)$ has no correspondence by the $\eta_{r}\left(\xi_{1} \ldots \xi_{r}\right)$ according to (I.20).

Finally we evaluate the scalar product between two normalized Dyson functionals by means of the expansion (I.17). We obtain

$$
\begin{aligned}
& g_{k n}=\int d_{k}\left(t_{1}^{\prime} \ldots t_{k}{ }^{\prime} ; j\right) d_{n}\left(t_{1} \ldots t_{n} ; j\right) \mathrm{d} j=\sum_{r=1}^{k} \sum_{l=1}^{n} B_{k r}\left(t_{1}{ }^{\prime} \ldots t_{k}{ }^{\prime} ; \xi_{1} \ldots \xi_{r}\right) B_{n l}\left(t_{1} \ldots t_{n} ; \xi_{1}{ }^{\prime} \ldots \xi_{l}{ }^{\prime}\right) \mathrm{d} \xi_{1} \ldots \mathrm{d} \xi_{r} \mathrm{~d} \xi_{1}{ }^{\prime} . . \mathrm{d} \xi_{l}{ }^{\prime} \\
& \times \int J_{r}\left(\xi_{1} \ldots \xi_{r} ; j\right) J_{l}\left(\xi_{1}^{\prime} \ldots \xi_{l}^{\prime} ; j\right) \mathrm{d} j \\
& =\sum_{r=0}^{\min (k, n)} b_{k,(k-r) / 2} b_{n,(n-r) / 2} \frac{1}{k !} \frac{1}{n !} \sum_{\lambda_{1} . . \lambda_{k}=1}^{k} \sum_{\mu_{1} . . \mu_{n}=1}^{n} \prod_{\alpha=1}^{r} \delta\left(t_{\alpha}^{\prime}-t_{\mu \alpha}\right) \\
& \times \delta\left(t_{\lambda_{r+1}}^{\prime}-t_{\lambda_{r+2}}^{\prime}\right) \ldots \delta\left(t_{\lambda_{k-1}}^{\prime}-t_{\lambda k}^{\prime}\right) \delta\left(t_{\mu_{r+1}}-t_{\mu_{r+2}}\right) \ldots \delta\left(t_{\mu_{n-1}}-t_{\mu_{n}}\right) \text {. }
\end{aligned}
$$

\section{Appendix II}

In this part we discuss the spinorial Hermite and Dyson functionals and their integration. Form the general requirement of a covariant spinor description it is necessary to formulate basic polynomials in the form

$$
P_{n}(j):=\int p_{n}\left(\begin{array}{c}
x_{1} \ldots x_{n} \\
a_{1} \ldots \alpha_{n}
\end{array}\right) j_{a_{1}}\left(x_{1}\right) \ldots j_{a_{n}}\left(x_{n}\right) \mathrm{d} x_{1} \ldots \mathrm{d} x_{n}
$$

with the "spinorial", for the anharmonic oscillator commuting source functions $j_{1}(x)$ and $j_{2}(x)$. In the case of nonlinear spinortheory anticommuting functions $j_{i}(x)$ have to be used, i. e. they have to be

12 F. A. Berezin, The Method of Second Quantization, Academic Press, New York 1966. elements of a Grassmann algebra ${ }^{12}$. Observing the first definition (I.2) of the corresponding Hermite polynomials, from a naive standpoint one would seperate (II.1) into two polynomials for $j_{1}$ and $j_{2}$ and apply then the definition operation (I.2). This of course would be on the first glance a noncovariant operation. Fortunately there exists besides of (I.2) with (I.3) a completely covariant definition of the Hermitean polynomials which can be applied immediately to (II.1), giving

$$
H P_{n}(j):=\exp \left\{-\frac{1}{2} \sum_{\alpha} \int \frac{\delta^{2}}{\delta_{j \alpha}(x)^{2}} \mathrm{~d} x\right\} P_{n}(j) .
$$

In the Fermion case one should have used instead of

$$
\sum_{\alpha} \int \frac{\delta^{2}}{\delta j_{a}(x)} \mathrm{d} x
$$


the expression

$$
\int \frac{\delta}{\delta j_{\beta}(x)} \sigma_{\alpha \beta} \frac{\delta}{\delta j_{\alpha}(x)} \mathrm{d} x
$$

with the totally antisymmetric unit tensor $\sigma_{\alpha \beta}$. It can be shown that (II.2) is completely equivalent to the separated polynomials defined by $j_{1}$ and $j_{2}$ from (II.1). Choosing the expansion functions $p_{\mathrm{n}}$ to be

$p_{n}\left(\begin{array}{c}t_{1} \ldots t_{n} ; x_{1} \ldots x_{n} \\ a_{1} \ldots a_{n} ; \beta_{1} \ldots \beta_{n}\end{array}\right):$

$$
=\frac{1}{n !} P \sum_{\lambda_{1}, \ldots \lambda_{n}=1}^{n} \prod_{i=1}^{n} \delta\left(t_{\lambda_{i}}-x_{i}\right) \delta_{\alpha_{\lambda_{i}}, \beta_{i}}
$$

we obtain the special set of polynomials

$$
P_{n}\left(\begin{array}{cc}
t_{1} \ldots t_{n} \\
a_{1} \ldots & a_{n}
\end{array}\right):=j_{\alpha_{1}}\left(t_{1}\right) \ldots j_{\alpha_{n}}\left(t_{n}\right)
$$

and the corresponding set of Hermite functionals is given by

paper, especially in Chapter 3. For completeness we

$$
\begin{aligned}
& J_{n}\left(\begin{array}{c}
t_{1} \ldots t_{n} \\
\alpha_{1} \ldots \alpha_{n}
\end{array} ; j\right)=\frac{1}{V n(1) ! \sqrt{n(2) !}} \\
& H P_{n}\left(\begin{array}{c}
t_{1} \ldots t_{n} \\
a_{1} \ldots \alpha_{n}
\end{array} ; j\right) \exp \left\{-\frac{1}{4} \sum_{\alpha} \int j_{\alpha}{ }^{2}(x) \mathrm{d} x\right\}
\end{aligned}
$$

where we define

$$
n(\alpha):=\sum_{i=1}^{n} \delta_{\alpha_{l}, \alpha} \quad(\alpha=1,2)
$$

It is easy to see, that one may define supervariables $r \equiv t, \alpha$, and obtains in this way

$$
\begin{aligned}
J_{n}\left(\mathfrak{x}_{1} \ldots \mathfrak{x}_{n} ; j\right)= & \frac{1}{\sqrt{n !}} H P_{n}\left(\mathfrak{x}_{1} \ldots \mathfrak{x}_{n} ; j\right) \\
& \exp \left\{-\frac{1}{4} \int j^{2}(\mathfrak{d}) \mathrm{dr}\right.
\end{aligned}
$$

but this description is completely equivalent to Appendix I. Therefore all formulas of Appendix I are valid for supervariables. The $n$ ! is then a shorthand notation for $n(1) ! n(2)$ ! and is often used in our

$$
\int J_{n}\left(\begin{array}{l}
t_{1} \ldots t_{n} \\
\left.\alpha_{1} \ldots \alpha_{n} ; j\right)
\end{array}\right) J_{m}\left(\begin{array}{l}
t_{1}^{\prime}, \ldots t_{m}^{\prime} \\
\alpha_{1}^{\prime} \ldots \alpha_{m}^{\prime} ; j
\end{array}\right) \mathrm{d} j=\delta_{n m} \frac{1}{m !} P \sum_{\lambda_{1} . . \lambda_{m}=1}^{m} \prod_{i=1}^{m} \delta\left(t_{\lambda_{i}}-t_{i}^{\prime}\right) \delta_{\alpha_{\lambda_{i},}, \alpha_{i}{ }^{\prime}}
$$

and the recurrence relations in the spinorial case:

$$
\begin{aligned}
& j_{\alpha}(t) J_{n}\left(\begin{array}{l}
t_{1} \ldots t_{n} \\
\alpha_{1} \ldots \alpha_{n}
\end{array} ; j\right)=\sqrt{n(\alpha)+1} J_{n+1}\left(\begin{array}{c}
t_{1} \ldots t_{n} t \\
a_{1} \ldots \alpha_{n} \alpha
\end{array} ; j\right)+\frac{1}{\sqrt{n(\alpha)}} \sum_{l=1}^{n} \delta\left(t-t_{l}\right) \delta_{\alpha_{l}, \alpha} J_{n-1}\left(\begin{array}{ccc}
t_{1} \ldots t_{l-1} & t_{l+1} \ldots t_{n} ; j \\
a_{1} \ldots \alpha_{l-1} a_{l+1} \ldots a_{n}
\end{array}\right), \\
& \frac{\delta}{\delta j_{\alpha}(t)} J_{n}\left(\begin{array}{c}
t_{1} \ldots t_{n} \\
a_{1} \ldots a_{n}
\end{array} ; j\right)=\frac{1}{2} \frac{1}{\sqrt{n(\alpha)}} \sum_{l=1}^{n} \delta\left(t-t_{l}\right) \delta_{\alpha_{l}, \alpha} J_{n-1}\left(\begin{array}{l}
t_{1} \ldots t_{l-1} t_{l+1} \ldots t_{n} \\
a_{1} \ldots \alpha_{l-1} \alpha_{l+1} \ldots \alpha_{n}
\end{array} ; j\right)-\frac{1}{2} \sqrt{n(\alpha)+1} J_{n+1}\left(\begin{array}{l}
t_{1} \ldots t_{n} t \\
a_{1} \ldots \alpha_{n} \alpha
\end{array} ;\right)
\end{aligned}
$$

In our paper we have used some modified definitions of the normalized Dyson and Hermite functionals, which are

$$
\begin{gathered}
d_{n}\left(\begin{array}{l}
t_{1} \ldots t_{n} \\
a_{1} \ldots a_{n}
\end{array} ; j\right)=\frac{i^{n}}{n !} P_{n}\left(\begin{array}{l}
t_{1} \ldots t_{n} \\
x_{1} \ldots \alpha_{n}
\end{array} ; j\right) \exp \left\{-\frac{1}{4} \sum_{\alpha} \int j_{\alpha}{ }^{2}(x) \mathrm{d} x\right\}, \\
J_{n}\left(\begin{array}{l}
t_{1} \ldots t_{n} \\
a_{1} \ldots \alpha_{n}
\end{array} ; j\right)=\frac{i^{n}}{\sqrt{n(1)} ! \sqrt{n(2) !} H P_{n}\left(\begin{array}{l}
t_{1} \ldots t_{n} \\
x_{1} \ldots \alpha_{n}
\end{array} ; j\right) \exp \left\{-\sum_{\alpha} \frac{1}{4} \int j_{\alpha}{ }^{2}(x) \mathrm{d} x\right\} .}
\end{gathered}
$$

Therefore, in this case, the transformations formulas between $d_{n}$ and $J_{n}$ corresponding to (I.17) and (I.18) of Appendix I are somewhat altered. The coefficients $a_{n,(n-k) / 2}^{\prime}$ respectively $b_{n,(n-k) / 2}^{\prime}$ now read:

$$
a_{n,(n-k) / 2}^{\prime}=: \frac{i^{n}}{i^{k}} \frac{\sqrt{n !}}{\sqrt{n(1) !} \sqrt{n(2) !}} a_{n,(n-k) / 2}, \quad \text { (II.12) } \quad b_{n,(n-k) / 2}^{\prime}=: \frac{i^{k}}{i^{n}} \frac{\sqrt{k(1) !} \sqrt{k(2) !}}{\sqrt{k !}} b_{n,(n-k) / 2} .
$$

\section{Appendix III}

In this part we discuss the calculation of matrixelements in the Hermitean representation. It is advantageous not to calculate the matrixelements itself, but already their projections on an arbitrary state function. The elementary matrix elements are

$$
\sum_{n=1}^{\infty}\left\langle J_{k}\left|h_{\beta}(\xi)\right| J_{n}\right\rangle-\frac{\sqrt{k !}}{\sqrt{n !}} g_{n}=g_{k+1}\left(\begin{array}{l}
t_{1} \ldots t_{k} \\
a_{1} \ldots \alpha_{k} \beta
\end{array}\right)+\sum_{\lambda_{1}=1}^{k} \delta\left(t_{\lambda_{1}}-\xi\right) \delta_{\beta, \alpha_{\lambda_{1}}} g_{k-1}\left(\begin{array}{ll}
t_{\lambda_{2}} \ldots t_{\lambda_{k}} \\
\alpha_{\lambda_{2}} \ldots \alpha_{\lambda_{k}}
\end{array}\right)
$$

and

$$
\sum_{n=1}^{\infty}\left\langle J_{k}\left|\frac{\delta}{\delta h_{\beta}(\xi)}\right| J_{n}\right\rangle \frac{\sqrt{k !}}{\sqrt{n !}} g_{n}=\frac{1}{2} g_{k+1}\left(\begin{array}{l}
t_{1} \ldots t_{k} \xi \\
\alpha_{1} \ldots \alpha_{k} \beta
\end{array}\right)-\frac{1}{2} \sum_{\lambda_{1}=1}^{k} \delta\left(t_{\lambda_{1}}-\xi\right) \delta_{\beta, \alpha_{\lambda_{1}}} g_{k-1}\left(\begin{array}{l}
t_{\lambda_{2}} \ldots t_{\lambda_{k}} \\
a_{\lambda_{2}} \ldots \alpha_{\lambda_{k}}
\end{array}\right)
$$


following immediately from formulas (I.11) respectively the corresponding formulas for super indizes. Now any other matrixelement can be decomposed by introducing intermediate states. Consider for example the matrixelement

$$
\begin{aligned}
& \qquad \sum_{n=1}^{\infty}\left\langle J_{k}\left|h_{\beta_{2}}\left(\xi_{2}\right) \frac{\delta}{\delta h_{\beta_{1}}\left(\xi_{1}\right)}\right| J_{n}\right\rangle \frac{\sqrt{k !}}{\sqrt{n !}} g_{n}=\sum_{n, m=1}^{\infty}\left\langle J_{k}\left|h_{\beta_{2}}\left(\xi_{2}\right)\right| J_{m}\right\rangle \frac{\sqrt{k !}}{\sqrt{m !}}\left\langle J_{m}\left|\frac{\delta}{\delta h_{\beta_{1}}\left(\xi_{1}\right)}\right| J_{n}\right\rangle \frac{\sqrt{m !}}{\sqrt{n !}} g_{n} . \\
& \text { Defining now } \\
& \qquad \sum_{n=1}^{\infty}\left\langle J_{m}\left|\frac{\delta}{\delta h_{\beta_{1}}\left(\xi_{1}\right)}\right| J_{n}\right\rangle \frac{\sqrt{m !}}{\sqrt{n !}}=: f_{m}
\end{aligned}
$$

then we have in the first step

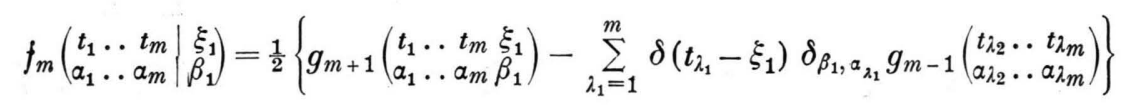

and in the second step

$$
\begin{aligned}
& \sum_{m=1}^{\infty}\left\langle J_{k}\left|h_{\beta_{2}}\left(\xi_{2}\right)\right| J_{m}\right\rangle \frac{\sqrt{k !}}{\sqrt{m !}} f_{m}=\frac{1}{2}\left\{g_{k+2}\left(\begin{array}{llll}
t_{1} \cdots & t_{m} & \xi_{1} & \xi_{2} \\
a_{1} \ldots & a_{m} & \beta_{1} & \beta_{2}
\end{array}\right)\right. \\
& +\sum_{\lambda_{1}=1}^{k}\left[\delta\left(t_{\lambda_{1}}-\xi_{2}\right) \delta_{\beta_{2}, a_{\lambda_{1}}} g_{k}\left(\begin{array}{l}
t_{\lambda_{2}} \ldots t_{\lambda_{k}} \\
a_{\lambda_{2}} \ldots \alpha_{\lambda_{k}} \beta_{1}
\end{array}\right)-\delta\left(t_{\lambda_{1}}-\xi_{1}\right) \delta_{\beta_{1}, a_{\lambda_{1}}} g_{k}\left(\begin{array}{l}
t_{\lambda_{2}} \ldots t_{\lambda_{k}} \\
a_{\lambda_{2}} \ldots a_{\lambda_{k}} \beta_{2}
\end{array}\right)\right] \\
& \left.-\delta\left(\xi_{1}-\xi_{2}\right) \delta_{\beta_{1}, \beta_{2}} g_{k}\left(\begin{array}{c}
t_{1} \ldots t_{k} \\
a_{1} \ldots a_{k}
\end{array}\right)-\sum_{\lambda_{1} \lambda_{2}=1}^{k} \delta\left(t_{\lambda_{1}}-\xi_{1}\right) \delta\left(t_{\lambda_{2}}-\xi_{2}\right) \delta_{\beta_{1}, a_{\lambda_{1}}} \delta_{\beta_{2}, a_{\lambda_{2}}} g_{k-2}\left(\begin{array}{l}
t_{\lambda_{3}} \ldots t_{\lambda_{k}} \\
a_{\lambda_{3}} \ldots a_{\lambda_{k}}
\end{array}\right)\right\} .
\end{aligned}
$$

Analogously all other elements can be evaluated.

From (III.6) follows easily the translational operator $P_{k l}$ of Section 6. First we have

$$
\int U_{\alpha \beta_{1}}\left(t-t_{1}\right) \frac{\mathrm{d}}{\mathrm{d} t} U_{\beta_{2} \alpha}^{-1}\left(t_{2}-t\right) \mathrm{d} t=\frac{1}{2 \pi} \int e^{-i p\left(t_{1}-t_{2}\right)}(-i p) \mathrm{d} p \delta_{\beta_{1}, \beta_{2}}=: \delta_{\beta_{1}, \beta_{2}} S\left(t_{1}-t_{2}\right)
$$

with

$$
S\left(t_{1}-t_{2}\right)=-S\left(t_{2}-t_{1}\right)
$$

i. e. $S(0)=O$. Then the translational operator reads according to (6.2)

$$
\sum_{l} P_{k l} \frac{\sqrt{k !}}{\sqrt{l !}} \eta_{l}=\sum_{l}\left\langle J_{k}\left|\int S\left(t_{1}-t_{2}\right) h_{\beta}\left(t_{1}\right) \frac{\delta}{\delta h_{\beta}\left(t_{2}\right)} \mathrm{d} t_{1} \mathrm{~d} t_{2}\right| J_{l}\right\rangle \frac{\sqrt{k !}}{\sqrt{l !}} \eta_{l} .
$$

Applying now formula (III.6) for the evaluation of (III.9) we have

$$
\int S\left(\xi_{1}-\xi_{2}\right) \eta_{k+2}\left(\begin{array}{cccc}
t_{1} \ldots t_{k} & \xi_{1} & \xi_{2} \\
a_{1} \ldots a_{k} & \beta & \beta
\end{array}\right) \mathrm{d} \xi_{1} \mathrm{~d} \xi_{2}=-\int S\left(\xi_{1}-\xi_{2}\right) \eta_{k+2}\left(\begin{array}{cccc}
t_{1} \ldots & t_{k} & \xi_{1} & \xi_{2} \\
a_{1} \ldots a_{k} & \beta & \beta
\end{array}\right) \mathrm{d} \xi_{1} \mathrm{~d} \xi_{2}=0 .
$$

Further it follows

$$
\sum_{\lambda_{1} \lambda_{2}=1}^{k} S\left(t_{\lambda_{1}}-t_{\lambda_{2}}\right) \delta_{\alpha_{\lambda_{1}}, \alpha_{\lambda_{2}}} \eta_{k-2}\left(\begin{array}{l}
t_{\lambda_{3}} \ldots t_{\lambda_{k}} \\
a_{\lambda_{3}} \ldots a_{\lambda_{k}}
\end{array}\right)=\sum_{\left(\lambda_{1} \lambda_{2}\right)=1}^{k}\left[S\left(t_{\lambda_{1}}-t_{\lambda_{2}}\right)+S\left(t_{\lambda_{2}}-t_{\lambda_{1}}\right)\right] \delta_{\alpha_{\lambda_{1}}, \alpha_{\lambda_{2}}} \eta_{k-2}\left(\begin{array}{l}
t_{\lambda_{3}} \ldots t t_{\lambda_{k}} \\
a_{\lambda_{3}} \ldots a_{\lambda_{k}}
\end{array}\right)=0
$$

because the bracket expressions vanishes. So we finally have

$$
\sum_{l=1}^{\infty} P_{k l} \eta^{l} \frac{\sqrt{k !}}{\sqrt{l !}}=\sum_{\lambda_{1}=1}^{k} \int S\left(t_{\lambda_{1}}-\xi\right) \eta_{k}\left(\begin{array}{c}
t_{\lambda_{2}} \ldots t_{\lambda_{k}} \xi \\
a_{\lambda_{2}} \ldots \alpha_{\lambda_{k}} \alpha_{\lambda_{1}}
\end{array}\right) \mathrm{d} \xi
$$

transforming this into Fourier space the formula (6.4) results.

\section{Appendix IV}

In this part the contraction formula of Section 5 are discussed explicitly. We therefore define the set of variables

$$
l_{k}^{m}:=\begin{aligned}
& q_{k+1} \ldots q_{2 m-k} \\
& a_{k+1} \ldots a_{2 m-k}
\end{aligned} .
$$

We then have especially for $k=0$ the original set $\quad l_{0}^{m}:=\frac{q_{1} \ldots q_{2 m}}{a_{1} \ldots \alpha_{2 m}}$. 
Furthermore in all fomulas we substitute instead of $q_{\lambda}$ simply $\lambda$ for abbreviation. Then by definition (5.1) the contraction for a symmetrical function $S_{2 m}\left(l_{0}^{m}\right)$ becomes

$$
P_{k} \ldots P_{1} S_{2 m}\left(l_{0}^{m}\right):=\int S_{2 m}\left(\begin{array}{ccccc}
q_{1}-\xi_{1} \ldots q_{k}-\xi_{k} & \xi_{1} \ldots & \xi_{k} & l^{m} \\
a_{1} & \ldots & a_{k} & a_{2 m} \ldots a_{2 m-k+1}
\end{array}\right) \mathrm{d} \xi_{1} \ldots \mathrm{d} \xi_{k}:=S_{2 m}^{k}\left(\begin{array}{ccc}
q_{1} \ldots & q_{k} \\
A_{1} \ldots A_{k}
\end{array} \mid l_{k}^{m}\right)
$$

with

$$
A_{j}:=\alpha_{j}, \alpha_{2 m-j+1}:=\alpha_{j}, \alpha_{j}^{\prime} .
$$

When we define the further set

$$
l_{k, \lambda_{1} \ldots \lambda_{i}}^{m}:=\left(\begin{array}{c}
q_{k+1} \ldots q_{k+\lambda_{1}-1} q_{k+\lambda_{1}+1} \ldots q_{k+\lambda_{i-1}} q_{k+\lambda_{i+1}} \ldots q_{2 m-k} \\
a_{k+1} \ldots \alpha_{k+\lambda_{1}-1} \alpha_{k+\lambda_{1}+1} \ldots \alpha_{k+\lambda_{i-1}} \alpha_{k+\lambda_{i+1}} \ldots \alpha_{2 m-k}
\end{array}\right), \quad \lambda_{1} \ldots \lambda_{i}:=q_{\lambda_{1}} \ldots q_{\lambda}
$$

the formulas to be contracted can be written like

$$
L_{i}^{0}(m)=: \sum_{\lambda_{1} . . \lambda_{i} \in l_{0}^{m}} g\left(\begin{array}{c}
\lambda_{1} \ldots \lambda_{i} \\
a_{\lambda_{1}} \ldots \alpha_{i}
\end{array}\right) S_{2 m-i}^{0}\left(l_{0, \lambda_{1} \ldots \lambda_{i}}^{m}\right)
$$

where $g\left(\lambda_{1} \ldots \lambda_{i}\right)$ is a symmetrical function in all arguments. In the dynamical equations $i=1 \ldots 4$ occurs. It is convenient to define further the following expressions

$$
L_{i}^{k}(m)=: \sum_{\lambda_{1} . . \lambda_{i} \in l_{k}^{m}} g\left(\begin{array}{c}
\lambda_{1} \ldots \lambda_{i} \\
a_{\lambda_{1}} \ldots a_{\lambda_{i}}
\end{array}\right) S_{2 m-i}^{k}\left(\begin{array}{c}
1 \ldots k \\
A_{1} \ldots A_{k}
\end{array} l_{k, \lambda_{1} \ldots \lambda_{i}}^{m}\right) .
$$

By this definition all operations of II, Appendix III can be overtaken for the spinorial case, with a slight modification: when variables of $g$ and $S$ are combined by integration one has to symmetrisize the spinor indices. For $i=1,2$ the formulae now read

$$
\begin{aligned}
& P_{k} \ldots P_{1} L_{1}^{0}(m)=L_{1}^{k}(m)+\sum_{\mu_{1}=1}^{k} \underset{\alpha_{\mu_{1}, \alpha_{\mu_{1}}}}{\operatorname{sym}} \int g\left(\begin{array}{c}
\mu_{1}-\xi \\
\alpha_{\mu_{1}}
\end{array}\right) S_{2 m-1}^{k-1}\left(\begin{array}{c}
\mu_{2} \ldots \mu_{k} \\
A_{\mu_{2}} \ldots A_{\mu_{k}}
\end{array} \mid \begin{array}{c}
\xi \\
\alpha_{\mu_{2}}
\end{array} l_{k}^{m}\right) \frac{\mathrm{d} \xi}{2 \pi}, \\
& P_{k} \ldots P_{1} L_{2}^{0}(m)=L_{2}^{k}(m)+\sum_{\mu_{1}=1}^{k} g^{1}\left(\begin{array}{c}
\mu_{1} \\
A_{\mu_{1}}
\end{array}\right) S_{2 m-2}^{k-1}\left(\begin{array}{c}
\mu_{2} \ldots \mu_{k} \\
A_{\mu_{2}} \ldots A_{\mu k}
\end{array} \mid l_{k}^{m}\right) \\
& +\sum_{\lambda_{1} \in l_{k}^{m}} \sum_{\mu_{1}=1}^{k} \operatorname{sym}_{\alpha_{\mu_{1}, \alpha_{\mu_{1}}}} \int g\left(\begin{array}{cc|c}
\mu_{1}-\xi, & \lambda_{1} \\
\alpha_{\mu_{1}} & \alpha_{\lambda_{1}}
\end{array}\right) S_{2 m-2}^{k-1}\left(\begin{array}{c}
\mu_{2} \ldots \mu_{k} \\
A_{\mu_{2}} \ldots A_{\mu_{k}}
\end{array} \mid \begin{array}{c}
\xi \\
\alpha_{\mu_{1}}
\end{array} l_{k, \lambda_{1}}^{m}\right) \frac{\mathrm{d} \xi}{2 \pi}
\end{aligned}
$$

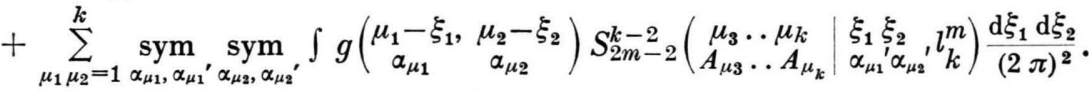

